\title{
Gravitational potential and X-ray luminosities of early-type galaxies observed with XMM-Newton and Chandra ${ }^{\star}$
}

\begin{abstract}
R. Nagino and K. Matsushita
Tokyo University of Science, 1-3 Kagurazaka, Shinjyuku-ku, 162-8601 Tokyo, Japan

e-mail: j1207705@ed.kagu.tus.ac.jp; matusita@rs.kagu.tus.ac.jp

Received 17 September 2008 / Accepted 25 March 2009

\section{ABSTRACT}

Aims. We study the dark matter content in early-type galaxies and investigate whether X-ray luminosities of early-type galaxies are determined by the surrounding gravitational potential.

Methods. We derived gravitational mass profiles of 22 early-type galaxies observed with XMM-Newton and Chandra.

Results. Sixteen galaxies show constant or decreasing radial temperature profiles, and their X-ray luminosities are consistent with kinematical energy input from stellar mass loss. The temperature profiles of the other 6 galaxies increase with radius, and their X-ray luminosities are significantly higher. The integrated mass-to-light ratio of each galaxy is constant at that of stars within $0.5-1 r_{\mathrm{e}}$, and increases with radius, where $r_{\mathrm{e}}$ is the effective radius of a galaxy. The scatter of the central mass-to-light ratio of galaxies was less in $K$-band light. At $3 r_{\mathrm{e}}$, the integrated mass-to-light ratios of galaxies with flat or decreasing temperature profiles are twice the value at $0.5 r_{\mathrm{e}}$, where the stellar mass dominates, and at $6 r_{\mathrm{e}}$, these increase to three times the value at $0.5 r_{\mathrm{e}}$.

Conclusions. This feature should reflect common dark and stellar mass distributions in early-type galaxies: within $3 r_{\mathrm{e}}$, the mass of dark matter is similar to the stellar mass, while within $6 r_{\mathrm{e}}$, the former is larger than the latter by a factor of two. In contrast, X-ray luminous galaxies have higher gravitational mass in the outer regions than X-ray faint galaxies. We describe these X-ray luminous galaxies as the central objects of large potential structures; the presence or absence of this potential is the main source of the large scatter in the X-ray luminosity.
\end{abstract}

Key words. galaxies: elliptical and lenticular, cD - galaxies: ISM - X-rays: galaxies - X-rays: ISM

\section{Introduction}

The bottom-up hierarchical theory of galaxy formation predicts that galaxies should be embedded in massive dark matter halos (e.g. Navarro et al. 1997). The presence of dark matter in spiral galaxies has been revealed through observations of stellar rotation curves (Rubin et al. 1978; van Albada et al. 1985). However, the study of halos in early-type galaxies is limited due to a lack of suitable and easy tracers such as rotation curves. Recently, observations of stellar velocity dispersion of early-type galaxies have reached to 1-2 $r_{\mathrm{e}}$, and a correlation between mass-to-light ratio and optical luminosity was found (e.g., Kronawitter et al. 2000; Gerhard et al. 2001). Here, $r_{\mathrm{e}}$ is the effective radius of a galaxy. For a small number of galaxies, mass profiles up to several $r_{\mathrm{e}}$ have been obtained using test particles such as globular clusters or planetary nebulae (e.g., Romanowsky et al. 2003; Chakrabarty \& Raychaudhury 2008).

X-ray observations provide a powerful tool to study the shape of the gravitational potential, and hence dark matter distributions, of early-type galaxies. These galaxies have a hot, $\mathrm{X}$-ray-emitting interstellar medium (ISM), which is considered to be gravitationally confined (e.g., Forman et al. 1985; Matsushita 2001; Fukazawa et al. 2006). The ISM luminosities of early-type galaxies vary by two orders of magnitude for the same optical $B$-band luminosity $\left(L_{B}\right)$ (e.g., Canizares et al. 1987; Beuing et al. 1999; Matsushita et al. 2000; Matsushita 2001),

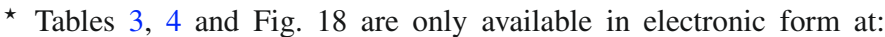
http://www . aanda.org whereas optical observations indicate that these galaxies are dynamically uniform systems (Djorgovski \& Davis 1987; Bender et al. 1993; Kronawitter et al. 2000). A key to solving this discrepancy is the extended X-ray emissions that have been detected around X-ray luminous early-type galaxies (Matsushita et al. 1998; Matsushita 2001). On the basis of ROSAT data, Matsushita (2001) discovered that, for most early-type galaxies, ISM luminosities within the optical radius agree with the kinematical energy input from the stellar mass loss $\left(L_{\sigma}\right)$. These galaxies have flat or decreasing temperature profiles against radius. In contrast, galaxies with ISM luminosities much larger than $L_{\sigma}$ show largely extended emission with a radius of a few tens of $r_{\mathrm{e}}$ with positive temperature gradients. XMM-Newton RGS observations provided evidence of a weak positive temperature gradient in the inner region of the ISM in NGC 4636, which has a much higher ISM luminosity than $L_{\sigma}$ (Xu et al. 2002). The correlation between the temperature gradient and spatial distribution was confirmed with Chandra observations (Fukazawa et al. 2006). These features suggest that X-ray luminous earlytype galaxies commonly sit in the center of a large-scale (a few hundred kpc) potential well, which leads to their high luminosities. Other galaxies may lack such a large-scale potential. On the basis of the extent of the ISM brightness, Matsushita (2001) denoted galaxies as either X-ray extended galaxies or X-ray compact galaxies. The gravitational mass profile of $\mathrm{cD}$ galaxies also shows two distinct contributions that can be assigned to the gravitational potential of the $\mathrm{cD}$ galaxy and that of the cluster (Matsushita et al. 2002). Thus, the only way to measure the 
Table 1. Galaxy sample in the XMM-Newton archive data.

\begin{tabular}{lccccccl}
\hline \hline Galaxy & Type $^{a}$ & $\begin{array}{c}D^{b} \\
(\mathrm{Mpc})\end{array}$ & $\begin{array}{c}r_{\mathrm{e}}^{c} \\
(\operatorname{arcmin})\end{array}$ & $\begin{array}{c}\log L_{B}{ }^{d} \\
\left(L_{\odot}\right)\end{array}$ & $\begin{array}{c}\sigma^{e} \\
(\mathrm{~km} / \mathrm{s})\end{array}$ & $\begin{array}{c}N_{\mathrm{H}}{ }^{f} \\
\left(10^{20} \mathrm{~cm}^{-2}\right)\end{array}$ & Note \\
\hline IC 1459 & -5.0 & 20.0 & 0.58 & 10.42 & 311 & 1.18 & $X_{\mathrm{C}}$ \\
NGC 720 & -5.0 & 20.3 & 0.60 & 10.34 & 240 & 1.54 & $X_{\mathrm{C}}$ \\
NGC 1316 & -2.0 & 16.9 & 1.35 & 10.78 & 250 & 1.89 & $X_{\mathrm{C}}$, Fornax \\
NGC 1332 & -2.0 & 17.7 & 0.47 & 10.22 & 319 & 2.23 & $X_{\mathrm{C}}$ \\
NGC 1395 & -5.0 & 20.0 & 0.81 & 10.33 & 254 & 1.99 & $X_{\mathrm{E}}$ \\
NGC 1399 & -5.0 & 16.9 & 0.68 & 10.31 & 362 & 1.34 & $X_{\mathrm{E}}$, Fornax \\
NGC 1549 & -5.0 & 13.4 & 0.78 & 10.10 & 213 & 1.46 & $X_{\mathrm{C}}$ \\
NGC 3585 & -5.0 & 21.6 & 0.60 & 10.56 & 227 & 5.58 & $X_{\mathrm{C}}$ \\
NGC 3607 & -2.0 & 19.9 & 0.73 & 10.41 & 223 & 1.48 & $X_{\mathrm{C}}$ \\
NGC 3665 & -2.0 & 32.4 & 0.48 & 10.54 & 186 & 2.06 & $X_{\mathrm{C}}$ \\
NGC 3923 & -5.0 & 25.8 & 0.83 & 10.68 & 241 & 6.21 & $X_{\mathrm{C}}$ \\
NGC 4365 & -5.0 & 16.8 & 0.83 & 10.40 & 266 & 1.62 & $X_{\mathrm{C}}$, Virgo \\
NGC 4382 & -2.0 & 16.8 & 0.91 & 10.64 & 187 & 2.52 & $X_{\mathrm{C}}$, Virgo \\
NGC 4472 & -5.0 & 16.8 & 1.74 & 10.92 & 302 & 1.66 & $X_{\mathrm{E}}$, Virgo \\
NGC 4477 & -2.0 & 16.8 & 0.63 & 10.14 & 175 & 2.64 & $X_{\mathrm{C}}$, Virgo \\
NGC 4526 & -2.0 & 16.8 & 0.74 & 10.41 & 260 & 1.65 & $X_{\mathrm{C}}$, Virgo \\
NGC 4552 & -5.0 & 16.8 & 0.49 & 10.35 & 262 & 2.57 & $X_{\mathrm{C}}$, Virgo \\
NGC 4636 & -5.0 & 17.0 & 1.48 & 10.46 & 208 & 1.81 & $X_{\mathrm{E}}$, Virgo \\
NGC 4649 & -5.0 & 16.8 & 1.15 & 10.74 & 343 & 2.20 & $X_{\mathrm{C}}$, Virgo \\
NGC 5044 & -5.0 & 38.9 & 0.89 & 10.60 & 237 & 4.93 & $X_{\mathrm{E}}$ \\
NGC 5322 & -5.0 & 31.6 & 0.56 & 10.86 & 233 & 1.81 & $X_{\mathrm{C}}$ \\
NGC 5846 & -5.0 & 28.5 & 1.05 & 10.66 & 251 & 4.26 & $X_{\mathrm{E}}$ \\
\hline
\end{tabular}

${ }^{a}$ Morphological type code from Tully (1988).

${ }^{b}$ Distance to the galaxy from Tully (1988).

${ }^{c}$ Effective radius from RC3 Catalog (de Vaucouleurs et al. 1991).

${ }^{d}$ Total $B$-band luminosity from Tully (1988).

${ }^{e}$ Central stellar velocity dispersion from Prugniel \& Simien (1996).

${ }^{f}$ Column density of the Galactic absorption from Dickey \& Lockman (1990).

gravitational mass profile of pure early-type galaxies is to observe the X-ray compact galaxies.

With ROSAT PSPC observations, O'Sullivan et al. (2003) found that the relation between the central stellar velocity dispersion and the temperature obtained from X-ray emission is similar to that for clusters and the relation between the X-ray luminosity and the temperature has a steep slope comparable to that found for galaxy groups.

Chandra and XMM-Newton have already observed several tens of early-type galaxies. Most of the analysis was done for $\mathrm{X}$-ray luminous and extended objects, and the number of X-ray compact galaxies with accurately derived gravitational mass profiles is still limited. For X-ray luminous galaxies, mass profiles are easily obtained over $10 r_{\mathrm{e}}$ (Fukazawa et al. 2006; Humphrey et al. 2006; Mathews et al. 2006). Using XMM-Newton, even for several X-ray compact galaxies, mass profiles can be derived up to several $r_{\mathrm{e}}$ (Fukazawa et al. 2006), and observed gravitational mass profiles of X-ray extended and X-ray compact galaxies are similar when plotted against radius in units of $r_{200}$. The dark matter profiles are well described by the NFW model (Navarro et al. 1996, 1997), which is based on numerical simulations assuming cold dark matter (CDM) as well as galaxy clusters (Fukazawa et al. 2006; Zappacosta et al. 2006). Chandra observations suggested that the shape of the X-ray isophotes is unrelated to the shape of the gravitational potential (Diehl \& Statler 2007, 2008).

In this study, we obtained gravitational mass profiles of 22 early-type galaxies observed with XMM-Newton and Chandra to investigate whether X-ray luminosities of early-type galaxies are determined by the surrounding gravitational potential and to study dark matter content in early-type galaxies. Throughout this paper, we adopt the solar abundances of Anders \& Grevesse (1989). Unless otherwise specified, errors are quoted at $1 \sigma$ confidence.

\section{Targets and observations}

We analyzed archival data of 22 early-type galaxies with distances less than $40 \mathrm{Mpc}$ and $B$-band luminosities $L_{B}>10^{10.1} L_{\odot}$ observed with XMM-Newton. The values of $L_{B}$ and the distances to the galaxies are taken from Tully (1988). The characteristics and observational log of the sample galaxies are summarized in Tables 1 and 2, respectively. The sample includes 15 elliptical and 7 S0 galaxies. Eight are located in the Virgo Cluster, 2 are in the Fornax Cluster, and the others are either in the field or in small groups. All observations were carried out with MOS1, MOS2, and PN together.

We also used Chandra data for 19 of the sample galaxies with good signal-to-noise ratios to derive the mass profile at their central regions. As summarized in Table 2, 15 galaxies were observed with ACIS-S, and 4 galaxies were observed with ACIS-I.

\section{Data reduction}

\subsection{XMM-Newton}

We analyzed MOS1, MOS2, and PN data of 21 galaxies. For NGC 4472, only MOS1 and MOS2 data were used, since PN data for this galaxy did not exist in the archive. We used XMMSAS version 7.0.0 for the data reduction.

We selected events with FLAG $=0$ and PATTERN smaller than 4 and 12 for the PN and MOS, respectively. A significant fraction of XMM-Newton observations is contaminated by soft proton flares. To filter the flares, for each observation, we made a count rate histogram of each detector, fitted the histogram with a Gaussian, and selected times within $2.5 \sigma$ of the mean of the histogram. The total exposure times after screening the flare events are summarized in Table 2. 
Table 2. Observational log of the sample galaxies.

\begin{tabular}{|c|c|c|c|c|c|c|}
\hline \multirow[b]{2}{*}{ Galaxy } & \multicolumn{3}{|c|}{ XMM-Newton } & \multicolumn{3}{|c|}{ Chandra } \\
\hline & $\operatorname{ObsID}^{a}$ & $\begin{array}{l}\text { exposure }^{b} \\
(\mathrm{ks})\end{array}$ & $\begin{array}{l}\text { total counts }{ }^{c} \\
\quad\left(\times 10^{3}\right)\end{array}$ & $\operatorname{ObsID}^{a}$ & ACIS & $\begin{array}{l}\text { exposure } \\
(\mathrm{ks})\end{array}$ \\
\hline IC 1459 & 0135980201 & $25,26,21$ & $8.9,14$ & 2196 & ACIS-S & 54 \\
\hline NGC 720 & 0112300101 & $17,18,11$ & $5.6,7.1$ & 492 & ACIS-S & 38 \\
\hline NGC 1316 & 0302780101 & $51,62,29$ & 32,38 & 2022 & ACIS-S & 26 \\
\hline NGC 1332 & 0304190101 & $54,54,41$ & 10,16 & 4372 & ACIS-S & 54 \\
\hline NGC 1395 & 0305930101 & $43,46,31$ & 15,21 & 799 & ACIS-I & 15 \\
\hline NGC 1399 & 0012830101 & $3,3,3$ & $7.1,10$ & 240 & ACIS-S & 43 \\
\hline NGC 1549 & 0205090201 & $8,8,6$ & $0.4,1.0$ & - & - & - \\
\hline NGC 3585 & 0071340201 & $11,11,7$ & $0.6,0.9$ & 2078 & ACIS-S & 35 \\
\hline NGC 3607 & 0099030101 & $14,14,6$ & $2.5,1.9$ & 2073 & ACIS-I & 38 \\
\hline NGC 3665 & 0052140201 & $23,24,19$ & $2.3,4.2$ & 3222 & ACIS-I & 18 \\
\hline NGC 3923 & 0027340101 & $32,32,24$ & 11,18 & 1563 & ACIS-S & 19 \\
\hline NGC 4365 & 0205090101 & $25,25,21$ & $3.8,7.1$ & 5923 & ACIS-S & 37 \\
\hline NGC 4382 & 0201670101 & $16,16,14$ & $3.0,5.6$ & 2016 & ACIS-S & 39 \\
\hline NGC 4472 & 0200130101 & $79,80,-$ & $300,-$ & 321 & ACIS-S & 37 \\
\hline NGC 4477 & 0112552101 & $13,13,7$ & $1.7,1.9$ & - & - & - \\
\hline NGC 4526 & 0205010201 & $20,20,16$ & $1.7,3.0$ & 3925 & ACIS-S & 41 \\
\hline NGC 4552 & 0141570101 & $21,22,15$ & 10,15 & 2072 & ACIS-S & 53 \\
\hline NGC 4636 & 0111190701 & $58,58,50$ & 210,320 & 4415 & ACIS-I & 73 \\
\hline NGC 4649 & 0021540201 & $46,46,36$ & 84,120 & 785 & ACIS-S & 31 \\
\hline NGC 5044 & 0037950101 & $17,17,8$ & 130,92 & 3225 & ACIS-S & 82 \\
\hline NGC 5322 & 0071340501 & $16,15,12$ & $1.0,1.5$ & - & - & - \\
\hline NGC 5846 & 0021540501 & $13,13,9$ & 25,28 & 788 & ACIS-S & 24 \\
\hline
\end{tabular}

${ }^{a}$ Observation number of the XMM-Newton and Chandra data.

${ }^{b}$ Exposure time of the EPIC-MOS1, MOS2, and PN, respectively.

${ }^{c}$ Total counts within $4 r_{\mathrm{e}}$ at $0.3-2.0 \mathrm{keV}$ of the MOS (MOS1 + MOS2) and PM, respectively.

The spectra were accumulated within rings centered on the center of each galaxy. Hereafter, we denote $r$ as the projected radius from the galaxy center. We excluded point sources with the sum of MOS1 and MOS2 count rates larger than 0.01 count/s. The edetect_chain command was used to detect point sources. The response matrix file and the auxiliary response file corresponding to each spectrum were calculated using SAS version 7.0.0.

The background spectrum was calculated for each spectrum by integrating blank sky data in the same detector regions. Among deep sky observations with the XMM, we selected data with the most similar background to that of each galaxy, after screening background flare events in the same way. Each background agrees well with the data at higher energies, as shown in Fig. 1 for NGC 4636.

Table 2 also summarizes total counts of MOS (MOS1+ MOS2) and PN within $4 r_{\mathrm{e}}$ centered on each galaxy. Here, an annular region, 10-14', from the center of each galaxy was used as a background after subtracting a blank sky data. The total counts of sum of those of MOS and PN have a wide range from 1000 to 600000 . We analyzed projected annular spectra of all of the sample galaxies, while fittings of deprojected spectra were performed for thirteen galaxies with the total counts $>12000$.

\subsection{Chandra}

Chandra data analysis was performed with the CIAO software package, version 3.3. We excluded time regions with a high background rate. We also eliminated point sources identified with the tool wavedetect. The outer region of each data set was subtracted as background.

\section{Spectral analysis and results}

\subsection{Spectral fit}

\subsubsection{Projected annular spectra}

To derive the gravitational mass profiles of individual galaxies, we need temperature and density profiles of the ISM. First, we fitted projected annular spectra centered on each galaxy from MOS and PN simultaneously, except NGC 4472. To exclude possible emission from our Galaxy and surrounding clusters, we also subtracted the spectrum in an annular region, $10^{\prime}-14^{\prime}$, from the annular spectra of each galaxy. The fitting model is a sum of a vAPEC model (Smith et al. 2001) and a power-law model. The vAPEC model represents thin thermal emission from the ISM, and the power-law model represents the contribution from unresolved low-mass X-ray binaries (LMXBs), where we fixed the power-law index at 1.6. Chandra observations found that total spectra of discrete sources in early-type galaxies are well described with this power-law model (Blanton et al. 2001; Randall et al. 2004). The two components were subjected to a common absorption with fixed column density $\left(N_{\mathrm{H}}\right)$ at the Galactic value from Dickey \& Lockman (1990). We organized heavy element abundances into three groups: the $\alpha$-element $\mathrm{O}$ group (O, Ne, and $\mathrm{Mg}$ ), Si group ( $\mathrm{Si}$ and $\mathrm{S}$ ), and $\mathrm{Fe}$ group (Fe and $\mathrm{Ni}$ ). The abundances of the three elemental groups were allowed to vary. For the innermost region of IC 1459, we added a power-law model from the central nuclei found by Chandra (Fabbiano et al. 2003).

For the brightest galaxies, NGC 4472, NGC 4636, NGC 4649, and NGC 5044, whose total counts within $4 r_{\mathrm{e}}$ are greater than 200000 , we performed spectral fitting on each annular region. In order to derive accurate temperature profiles of the ISM in the other galaxies with lower signal-to-noise ratio, the spectra 


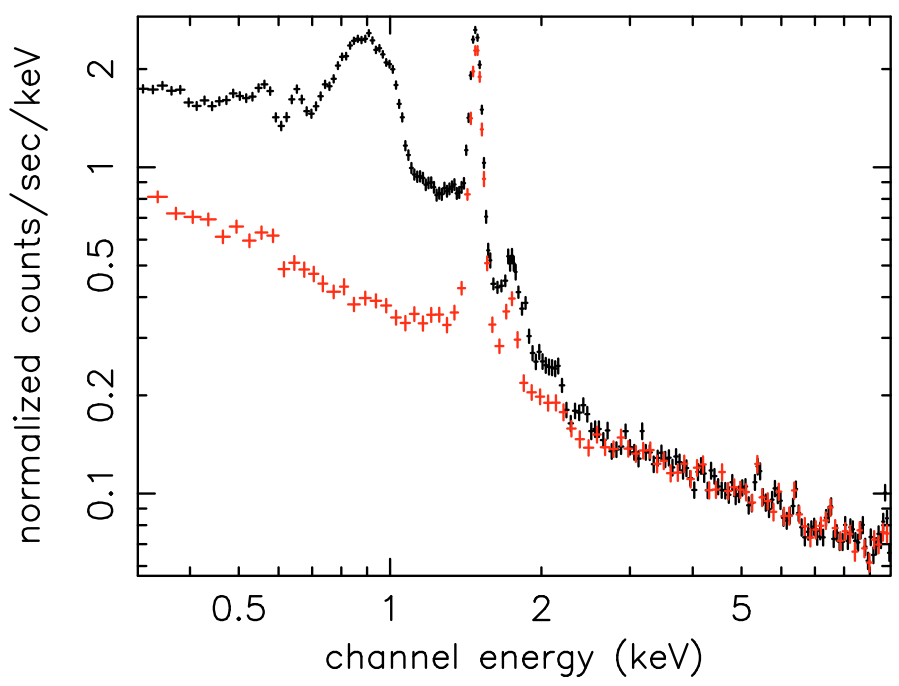

Fig. 1. Raw MOS (MOS1 + MOS2) spectrum at $r=6-14^{\prime}$ of NGC 4636 (black), and the adopted background spectrum (red).

of all annular regions were fitted simultaneously, where the ISM abundances were assumed to have common values.

Table 3 and Fig. 18 summarize the results of the spectral fitting, ISM temperature, abundance, and ISM luminosity. Figure 2 shows MOS and PN spectra of the innermost regions of several representative galaxies. The spectra of X-ray faint galaxies, whose total counts within $4 r_{\mathrm{e}}$ are less than 12000 counts, provide gradually smaller values of the reduced- $\chi^{2}$ with this singletemperature model (hereafter 1T model) for the ISM. However, in a representative spectrum of NGC 4382 whose ISM temperature is $\sim 0.4 \mathrm{keV}$, there are residual structures around $0.9 \mathrm{keV}$. The X-ray brighter galaxies show larger reduced- $\chi^{2}$. The spectra of galaxies with $k T \sim 0.6 \mathrm{keV}$, NGC 4636, NGC 720 and NGC 3923, show common residuals at $0.7-0.9 \mathrm{keV}$ (Fig. 2). NGC 4649, which is the galaxy with $k T \sim 0.8 \mathrm{keV}$, has different residual structures from the other three galaxies.

\subsubsection{Deprojected spectra}

To consider projection effects, we performed spectral fittings of deprojected spectra. For the data with high statistics, for which the total counts of the sum of those of MOS and PN within $4 r_{\mathrm{e}}$ are greater than 12000 , deprojected spectra were calculated using "onion peeling" methods by subtracting the contribution from the outer shell regions for all spectral components, assuming the ISM is spherically symmetric as described by Takahashi (2004). We then fitted the deprojected spectra with the 1T model in the same way as in Sect. 4.1.1.

Table 4 and Fig. 18 summarize the results. The reduced $\chi^{2}$ reduced to $\sim 1$ except at the innermost regions of three brightest galaxies, NGC 4472, NGC 4636, and NGC 4649. However, residual structures around $0.7-0.9 \mathrm{keV}$ in the projected spectra still remain in the deprojected spectra fitted with the $1 \mathrm{~T}$ model (Fig. 2). We also fitted the spectra of innermost regions of the three brightest galaxies with a two-temperature vAPEC model for the ISM (hereafter 2T model). The results are summarized in Table 4. The reduced $\chi^{2}$ reduced to $1.2-1.5$, and even the $2 \mathrm{~T}$ model gives similar residual structures (Fig. 2). These discrepancies in the Fe-L energy range are also seen in the RGS spectrum of the X-ray luminous elliptical galaxy, NGC 4636
(Xu et al. 2002). The Suzaku observations of NGC 720 and NGC 1404 whose ISM temperatures are also $\sim 0.6 \mathrm{keV}$ also give similar residual structures (Matsushita et al. 2007; Tawara et al. 2008). Therefore, these residual structures are likely to be related to poorly modeled $\mathrm{Fe}-\mathrm{L}$ lines.

The spectral fittings of the deprojected spectra mostly give the same temperatures as those of the projected ones (Fig. 18). Therefore, for the fainter galaxies for which the deprojected analysis were not performed, we used the temperature profiles derived from the projected spectra to derive gravitational mass profiles.

\subsection{Results}

\subsubsection{ISM luminosities within $4 r_{\mathrm{e}}$}

We derived the absorption-corrected ISM luminosities ( $\left.L_{\mathrm{ISM}}\right)$ and the luminosities of the power-law component $\left(L_{\text {hard }}\right)$ in the energy band of $0.3-2.0 \mathrm{keV}$ within $4 r_{\mathrm{e}}$ (Table 5). The values of $L_{\mathrm{ISM}}$ derived from the deprojected spectra are close to those from projected annular spectra. Hereafter, we use $L_{\mathrm{ISM}}$ from the deprojected analysis. For the X-ray fainter galaxies without deprojected analysis, we use the values derived from the projected analysis.

The relationship of $L_{\text {ISM }}$ to $L_{B}$ is shown in the left panel of Fig. 3. The sample galaxies have $L_{B}$ from $10^{10.1}$ to $10^{10.9} L_{\odot}$. On the other hand, $L_{\mathrm{ISM}}$ scatters from $10^{39.4} \mathrm{erg} / \mathrm{s}$ to $10^{42.4} \mathrm{erg} / \mathrm{s}$.

The right panel of Fig. 3 shows the correlation between $L_{\text {ISM }}$ within $4 r_{\mathrm{e}}$ and $L_{B} \sigma^{2}$, with $\sigma$ denoting the central stellar velocity dispersion in each galaxy. If stellar motion is the main heat source for the hot ISM, its X-ray luminosity should be approximated by the input rate of the kinetic energy of the gas from stellar mass loss. This is proportional to $L_{B} \sigma^{2}$, because the mass-loss rate is thought to be proportional to $L_{B}$ (e.g., Ciotti et al. 1991). Figure 3 also shows the expected energy input from stellar mass loss $\left(L_{\sigma}\right)$ assuming a mass-loss rate of $1.5 \times 10^{-11}\left(L_{B} / L_{\odot}\right) t_{15}^{-1.3} M_{\odot} \mathrm{yr}^{-1}$ (Ciotti et al. 1991). Here, $t_{15}$ is the stellar age in units of $15 \mathrm{Gyr}$, and we assumed a stellar age of 12 Gyr. Several galaxies have larger $L_{\mathrm{ISM}}$ than $L_{\sigma}$, while $L_{\mathrm{ISM}}$ values of the other galaxies are similar to or smaller than $L_{\sigma}$.

\subsubsection{Temperature profiles of the ISM and classification with X-ray extended and X-ray compact galaxies}

Figure 4 shows the derived radial temperature profiles of the ISM. Some galaxies have gradually increasing temperature profiles toward the outer radius. In contrast, other galaxies have flat or decreasing radial temperature profiles. The galaxies with positive temperature gradients have high ISM temperatures of $0.8-1.5 \mathrm{keV}$ at a radius of several times $r_{\mathrm{e}}$, which are comparable to those of galaxy groups. On the other hand, the temperatures of the other galaxies are systematically lower at $0.2-0.6 \mathrm{keV}$. The derived temperature profiles were fitted with the sum of a constant and single- or double- $\beta$ functions.

The derived temperature profiles are mostly consistent with previous results from ROSAT (Matsushita 2001) and Chandra (Fukazawa et al. 2006; Athey 2007), except central regions of several brightest galaxies with positive temperature gradients, due to the higher angular resolution of Chandra.

In Fig. 5, we plotted $k T\left(1-4 r_{\mathrm{e}}\right) / k T\left(<1 r_{\mathrm{e}}\right)$ and $k T\left(4-8 r_{\mathrm{e}}\right) / k T\left(<1 r_{\mathrm{e}}\right)$ against $L_{\mathrm{ISM}} / L_{B} \sigma^{2}$. Here, $k T\left(<1 r_{\mathrm{e}}\right)$, $k T\left(1-4 r_{\mathrm{e}}\right)$, and $k T\left(4-8 r_{\mathrm{e}}\right)$ correspond to the emission-weighted 

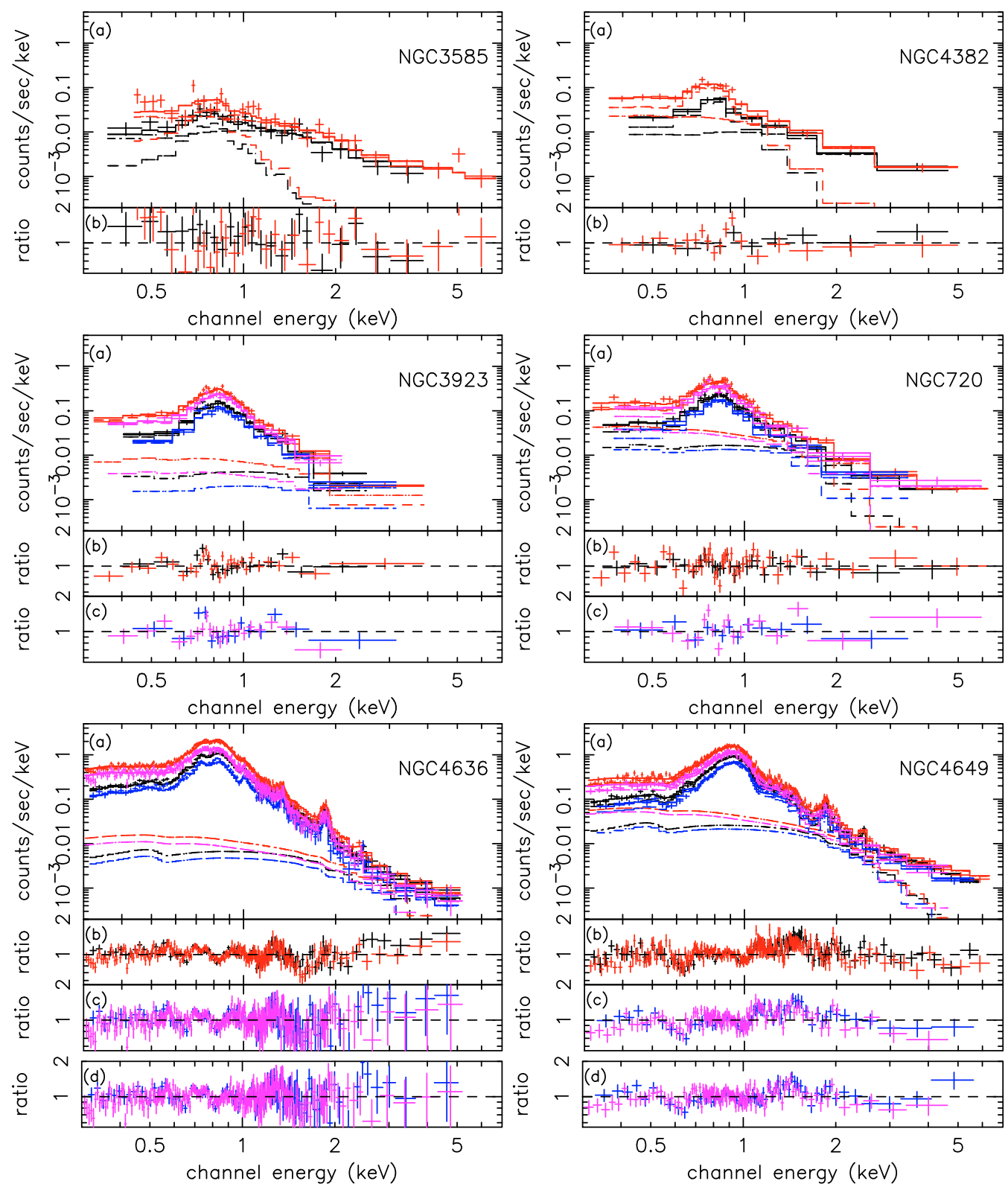

Fig. 2. a) Innermost projected spectra observed with MOS (black) and PN (red), and innermost deprojected spectra with MOS (blue) and PN (magenta). These spectra are fitted with a vAPEC model plus power-law multiplied by the Galactic absorption (solid line). Dashed lines correspond to the contribution from each component. b), c) The actual data to model ratio from the fit in panel a). The meanings of colors correspond to that in panel a). d) The same as panel b) and c), but using the vAPEC + vAPEC model plus power-law multiplied by the Galactic absorption as the fitting model.

ISM temperatures of regions in $R<1 r_{\mathrm{e}}, 1 r_{\mathrm{e}}<R<4 r_{\mathrm{e}}$ and $4 r_{\mathrm{e}}<R<8 r_{\mathrm{e}}$, respectively. Here, $R$ is the deprojected radius from the galaxy center. In general, the temperature profiles of galaxies with $L_{\mathrm{ISM}} \lesssim L_{\sigma}$ have smaller $k T\left(1-4 r_{\mathrm{e}}\right)$ and $k T\left(4-8 r_{\mathrm{e}}\right)$ than $k T\left(<1 r_{\mathrm{e}}\right)$. In contrast, $L_{\mathrm{ISM}}$ of galaxies with $k T\left(4-8 r_{\mathrm{e}}\right) / k T\left(<1 r_{\mathrm{e}}\right)>1.3$ are systematically larger than $L_{\sigma}$.

Our sample galaxies are divided into two types: X-ray faint galaxies with flat or negative temperature gradients and X-ray luminous galaxies with positive temperature gradients. The ISM luminosities in the former type are consistent with heating by stellar motion, while galaxies of the latter type need additional sources of heating. Hereafter, we denote galaxies with $k T\left(4-8 r_{\mathrm{e}}\right) / k T\left(<1 r_{\mathrm{e}}\right)>1.3$ as X-ray extended $\left(X_{\mathrm{E}}\right)$ galaxies and others as X-ray compact $\left(X_{\mathrm{C}}\right)$ galaxies. The classification of each galaxy is summarized in Table 1 .

\section{Spatial analysis and results}

\subsection{X-ray surface brightness and gas density profiles}

We derived radial profiles of X-ray surface brightness from background-subtracted and vignetting-corrected X-ray images from MOS1 and MOS2. We considered only photons in the energy band $0.8-2.0 \mathrm{keV}$, where the ISM emission dominates. PN data were not used for this analysis because of gaps between CCD chips. Figure 6 shows two representative X-ray surface brightness profiles, those of an $X_{\mathrm{E}}$ galaxy, NGC 4636, and an $X_{\mathrm{C}}$ galaxy, NGC 720 .

Assuming circular symmetry, we deprojected the X-ray surface brightness profiles to derive gas density profiles. In order to subtract emission from outside the field of view, we first fitted the radial surface brightness profile within $8 r_{\mathrm{e}}$ of each 

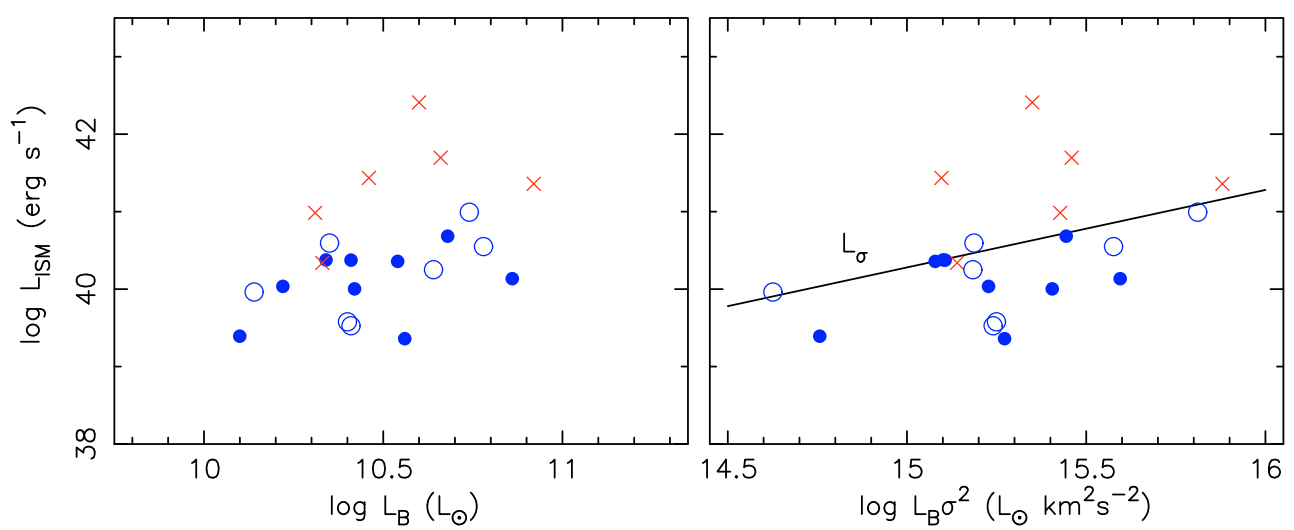

Fig. 3. $L_{\mathrm{ISM}}\left(<4 r_{\mathrm{e}}\right)$ of galaxies plotted against $L_{B}$ (left panel) and $L_{B} \sigma^{2}$ (right panel). Symbols indicate galaxy categories defined in Sect. 4.2 .2 for $X_{\mathrm{E}}$ galaxies (red crosses), field $X_{\mathrm{C}}$ galaxies (filled blue circles), and $X_{\mathrm{C}}$ galaxies in the clusters (open blue circles). The solid line represents the kinetic heating rate by stellar mass loss $\left(L_{\sigma}\right)$.

Table 5. X-ray luminosities within $4 r_{\mathrm{e}}$ of each sample galaxy.

\begin{tabular}{lccc}
\hline \hline Galaxy & $\begin{array}{c}\log L_{\text {ISM }}{ }^{a} \\
\text { projection } \\
\text { (erg/s) }\end{array}$ & $\begin{array}{c}\log L_{\text {hard }}{ }^{b} \\
\text { projection } \\
\text { (erg/s) }\end{array}$ & $\begin{array}{c}\log L_{\text {ISM }}{ }^{a} \\
\text { deprojection } \\
\text { (erg/s) }\end{array}$ \\
\hline IC 1459 & 40.08 & 40.28 & 40.00 \\
NGC 720 & 40.46 & 39.91 & 40.38 \\
NGC 1316 & 40.63 & 40.20 & 40.55 \\
NGC 1332 & 40.12 & 39.74 & 40.04 \\
NGC 1395 & 40.43 & 40.16 & 40.34 \\
NGC 1399 & 41.13 & 40.32 & 40.98 \\
NGC 1549 & 39.39 & 39.09 & - \\
NGC 3585 & 39.36 & 39.39 & - \\
NGC 3607 & 40.37 & 39.86 & - \\
NGC 3665 & 40.36 & 39.96 & - \\
NGC 3923 & 40.74 & 40.27 & 40.68 \\
NGC 4365 & 39.58 & 39.86 & - \\
NGC 4382 & 40.25 & 39.83 & - \\
NGC 4472 & 41.40 & 40.51 & 41.36 \\
NGC 4477 & 39.96 & 39.54 & - \\
NGC 4526 & 39.53 & 39.64 & - \\
NGC 4552 & 40.62 & 40.27 & 40.60 \\
NGC 4636 & 41.46 & 40.27 & 41.44 \\
NGC 4649 & 41.00 & 40.41 & 40.99 \\
NGC 5044 & 42.49 & 41.01 & 42.41 \\
NGC 5322 & 40.14 & 39.82 & - \\
NGC 5846 & 41.72 & 40.56 & 41.70 \\
\hline X-Tay 14719
\end{tabular}

a The X-ray luminosity of the thermal emission in the range of $0.3-2.0 \mathrm{keV}$ derived from the projected and deprojected spectra.

${ }^{b}$ The X-ray luminosity of the non thermal emission in the range of $0.3-2.0 \mathrm{keV}$ derived from the projected spectra.

galaxy and assumed that the profile extends outside the field of view. The radial profiles of most of the galaxies were fitted with a $\beta$-model. The brightness of several galaxies in clusters are clearly constant at the outer regions because of the surrounding intra-cluster medium (ICM). Therefore, we fitted these profiles with the sum of a $\beta$ model and a constant. Several X-ray luminous galaxies need a double- $\beta$ model to fit the surface brightness profiles. Because at $>5 r_{\mathrm{e}}$, one of the $\beta$ models dominates, the profiles at $r>5 r_{\mathrm{e}}$ are fitted with a single- $\beta$ model. The derived density profiles are summarized in Fig. 7. Since we need only the gradient of a density profile to derive a gravitational mass profile, the plotted density profiles were arbitrarily normalized.

We also used Chandra data for 19 galaxies with sufficiently high signal-to-noise ratios to derive accurate $\mathrm{X}$-ray surface brightness profiles within 1-2 $r_{\mathrm{e}}$. Radial profiles of X-ray surface brightness were derived from ACIS X-ray images in the energy band $0.3-2.0 \mathrm{keV}$. Then, we deprojected the X-ray surface brightness profiles and derived gas density profiles in the same way as for the XMM-Newton data. As summarized in Fig. 7, normalized density profiles of the ISM derived from XMMNewton and Chandra are mostly consistent with each other from $0.5 r_{\mathrm{e}}$ to $2 r_{\mathrm{e}}$.

We then fitted the derived gas density profiles of XMMNewton at $R>0.5 r_{\mathrm{e}}$ and that of Chandra within $R<2 r_{\mathrm{e}}$ of each galaxy simultaneously with a $\beta$ model, as $f(R)=S_{0}(1+$ $\left.\left(R / R_{\mathrm{c}}\right)^{2}\right)^{-\mathrm{IN}}$. Most of the density profiles were well fitted with this single- $\beta$ model (Fig. 7). The derived $R_{\mathrm{c}}$ values of galaxies with Chandra data are almost about $0.05 r_{\mathrm{e}}$. Therefore, we fixed the $R_{\mathrm{c}}$ value to $0.05 r_{\mathrm{e}}$ to fit the gas density profiles of galaxies without Chandra data. For NGC 4552 we also fixed the $R_{\mathrm{c}}$ value to $0.05 r_{\mathrm{e}}$, because of an edge-like structure at 0.4-0.6 $r_{\mathrm{e}}$ observed with Chandra (Machacek et al. 2006). Several X-ray luminous galaxies need a double- $\beta$ model to fit the density profiles.

The ISM and the hard component may have different surface brightness profiles. Therefore, we also derived gas density profiles of galaxies whose $L_{\text {ISM }}$ is less than $80 \%$ of the total luminosity within $4 r_{\mathrm{e}}$, directly from the normalization of the ISM component derived from spectral fittings. The best-fit $\beta$-model of the density profile of each galaxy is plotted in Fig. 18. The best-fit values of $\beta$ derived in this way are mostly consistent with those derived from surface brightness profiles, although several galaxies show discrepancies of a few tens of $\%$.

\section{Mass profiles}

We then calculated the total mass profile $M(R)$ within a threedimensional radius $R$ from the obtained best-fit functions of ISM temperature $T(R)$ and gas density $n(R)$ profiles from the surface brightness, assuming hydrostatic equilibrium and circular symmetry, by the equation

$$
M(R)=-\frac{k T(R) \cdot R}{G \mu m_{\mathrm{p}}}\left(\frac{\mathrm{d} \ln n(R)}{\mathrm{d} \ln R}+\frac{\mathrm{d} \ln T(R)}{\mathrm{d} \ln R}\right),
$$

where $m_{\mathrm{p}}$ is the proton mass, $k$ is the Boltzmann constant, $G$ is the constant of gravity, and $\mu \sim 0.62$ is the mean particle mass in units of $m_{\mathrm{p}}$. Figure 8 summarizes the derived mass profiles. For NGC 1549, NGC 4477, and NGC 5322, the mass profiles 

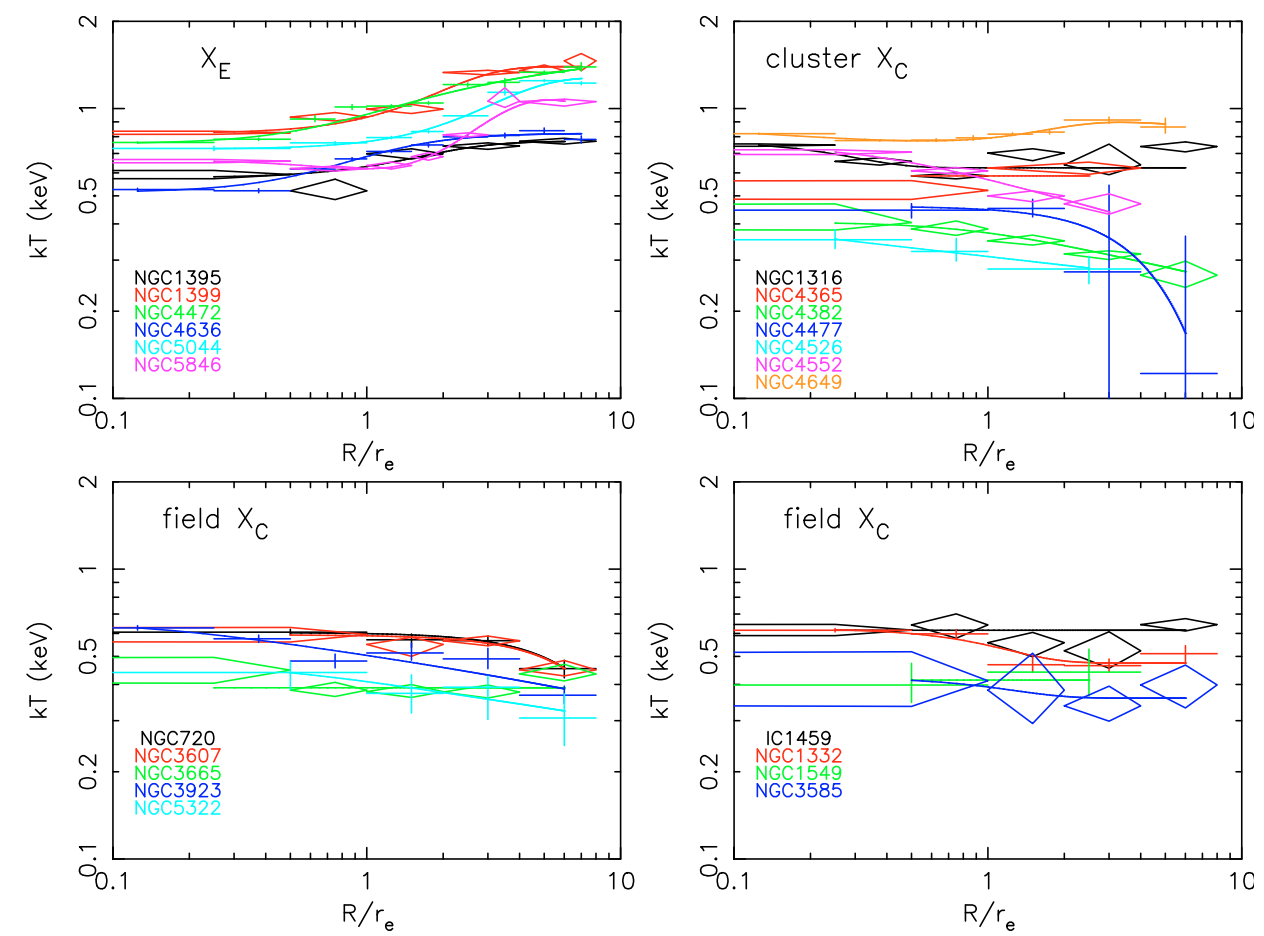

Fig. 4. The derived temperature profiles of the ISM. The $X_{\mathrm{E}}$ galaxies are plotted in the top left panel, the $X_{\mathrm{C}}$ galaxies in clusters are in the top right, and the field $X_{\mathrm{C}}$ galaxies are in the bottom panels. Colors indicate individual galaxies. The solid lines represent the best-fit function.
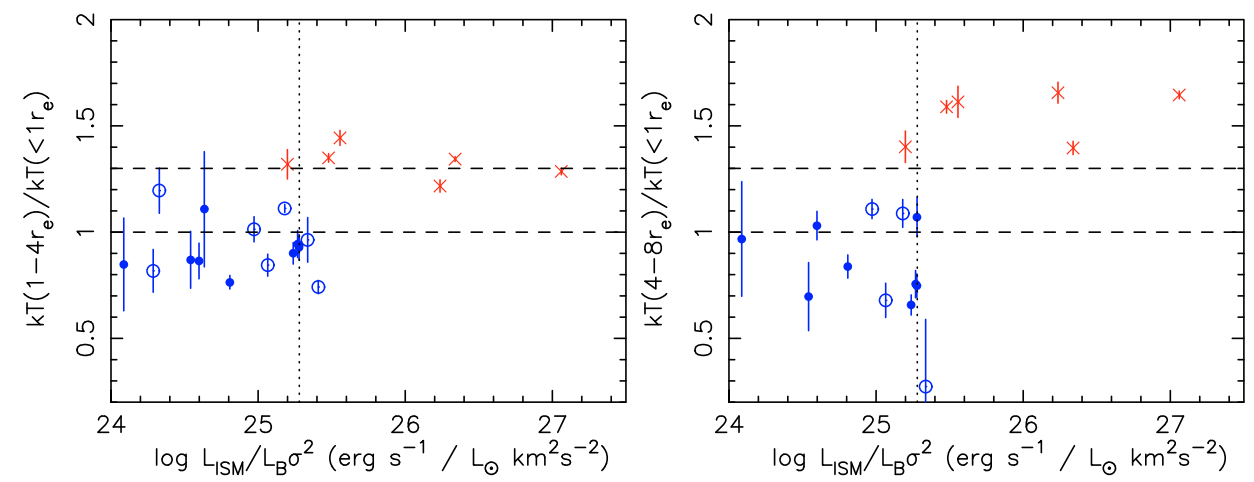

Fig. 5. The ISM temperature gradients parametrized by $k T\left(1-4 r_{\mathrm{e}}\right) / k T\left(<1 r_{\mathrm{e}}\right)\left(\right.$ left panel) and $k T\left(4-8 r_{\mathrm{e}}\right) / k T\left(<1 r_{\mathrm{e}}\right)($ right panel) plotted against $L_{\mathrm{ISM}} / L_{B} \sigma^{2}$. Meanings of the symbols are the same as those in Fig. 3. The dotted line represents the rate of kinetic heating by stellar mass loss. The dashed lines indicate that $k T\left(1-4 r_{\mathrm{e}}\right)$ (or $\left.k T\left(4-8 r_{\mathrm{e}}\right)\right)$ is 1 or 1.3 times higher than $k T\left(<1 r_{\mathrm{e}}\right)$.

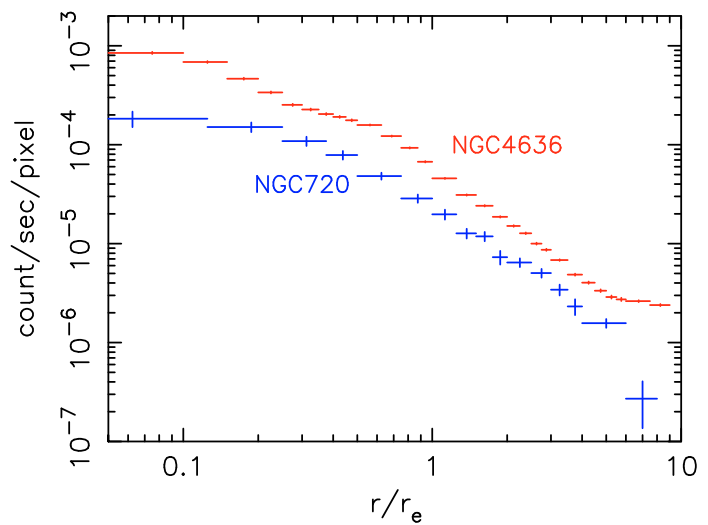

Fig. 6. X-ray surface brightness profiles derived from MOS images of NGC 720 (blue) and NGC 4636 (red).

within $0.5 r_{\mathrm{e}}$ were not plotted in the figure, since we used only XMM-Newton data for those galaxies.
In addition, the upper and lower limits of the mass profiles were calculated considering the errors in the temperature and the temperature and density gradients of each data bin. The upper and lower limits of the temperature and density gradients of the $i$ th shell were obtained from the ratio of the value within $i+1$ th shell to that within $i-1$ th shell. Here, we used the temperature profiles derived from XMM-Newton. At $R<1 r_{\mathrm{e}}$ and $R>1 r_{\mathrm{e}}$, density profiles derived from Chandra and XMM-Newton, respectively, were used. For NGC 4636, the density at $0.3-0.4 r_{\mathrm{e}}$ is significantly smaller than the best-fit function, due to the existence of complicated structure discovered by Chandra (Jones et al. 2002). Therefore, we ignored this shell when deriving the mass profiles. As summarized in Fig. 8, the total masses derived in this way are mostly consistent with those using the best-fit functions.

We also derived stellar mass profiles, using the deprojected de Vaucouleurs profile of Mellier \& Mathez (1987), assuming stellar $M / L_{B}$ is in the range from 3 to 8 in solar units. We plotted these profiles in Fig. 8. Within $1 r_{\mathrm{e}}$, the gravitational mass 

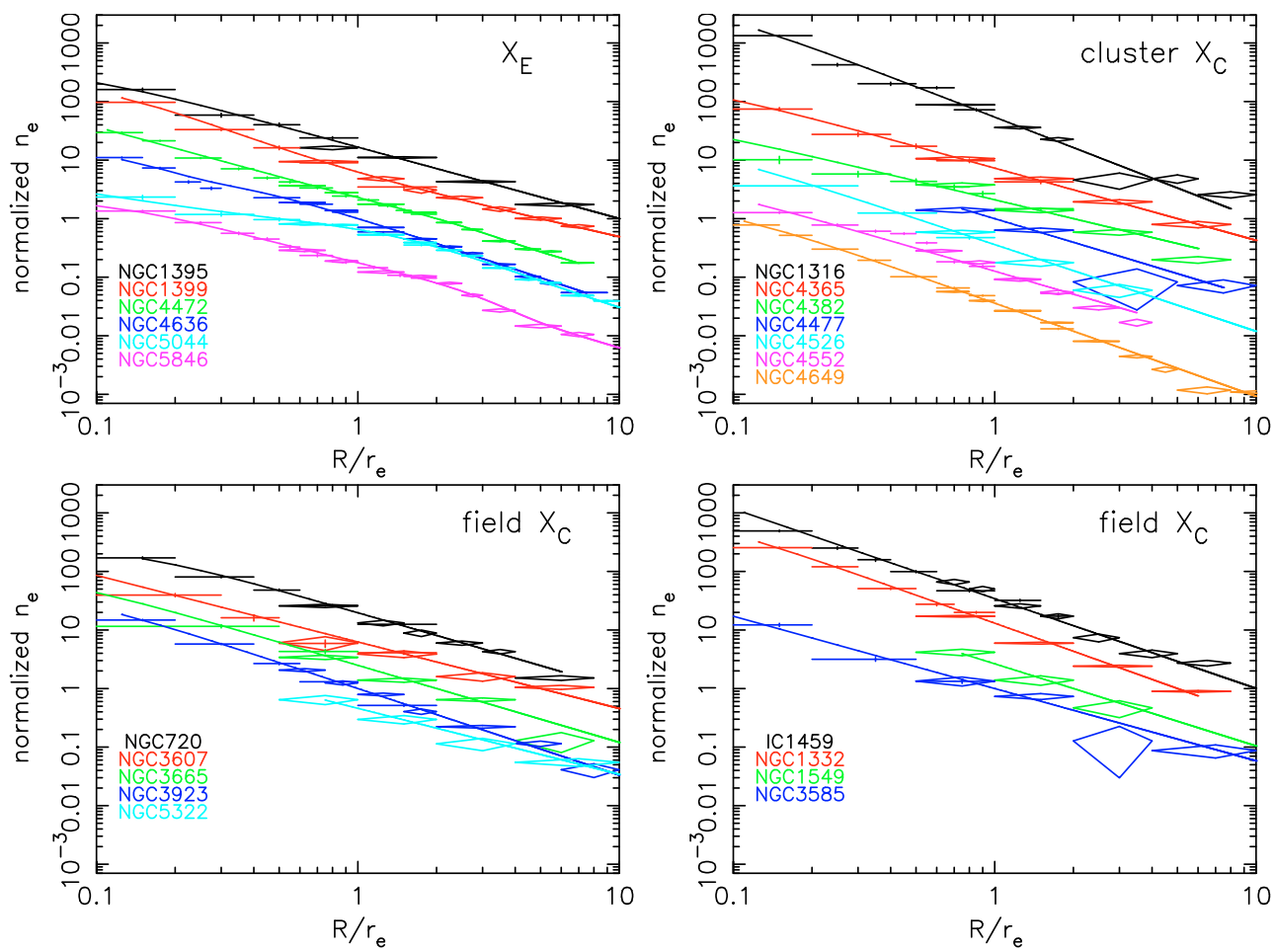

Fig. 7. ISM density profiles. The $X_{\mathrm{E}}$ galaxies are plotted in the top left panel, the $X_{\mathrm{C}}$ galaxies in clusters are in the top right, and the field $X_{\mathrm{C}}$ galaxies are in the bottom panels. These profiles are arbitrarily normalized. Colors indicate individual galaxies. Solid lines represent the best-fit function. Crosses and diamonds correspond to Chandra and XMM-Newton data, respectively.
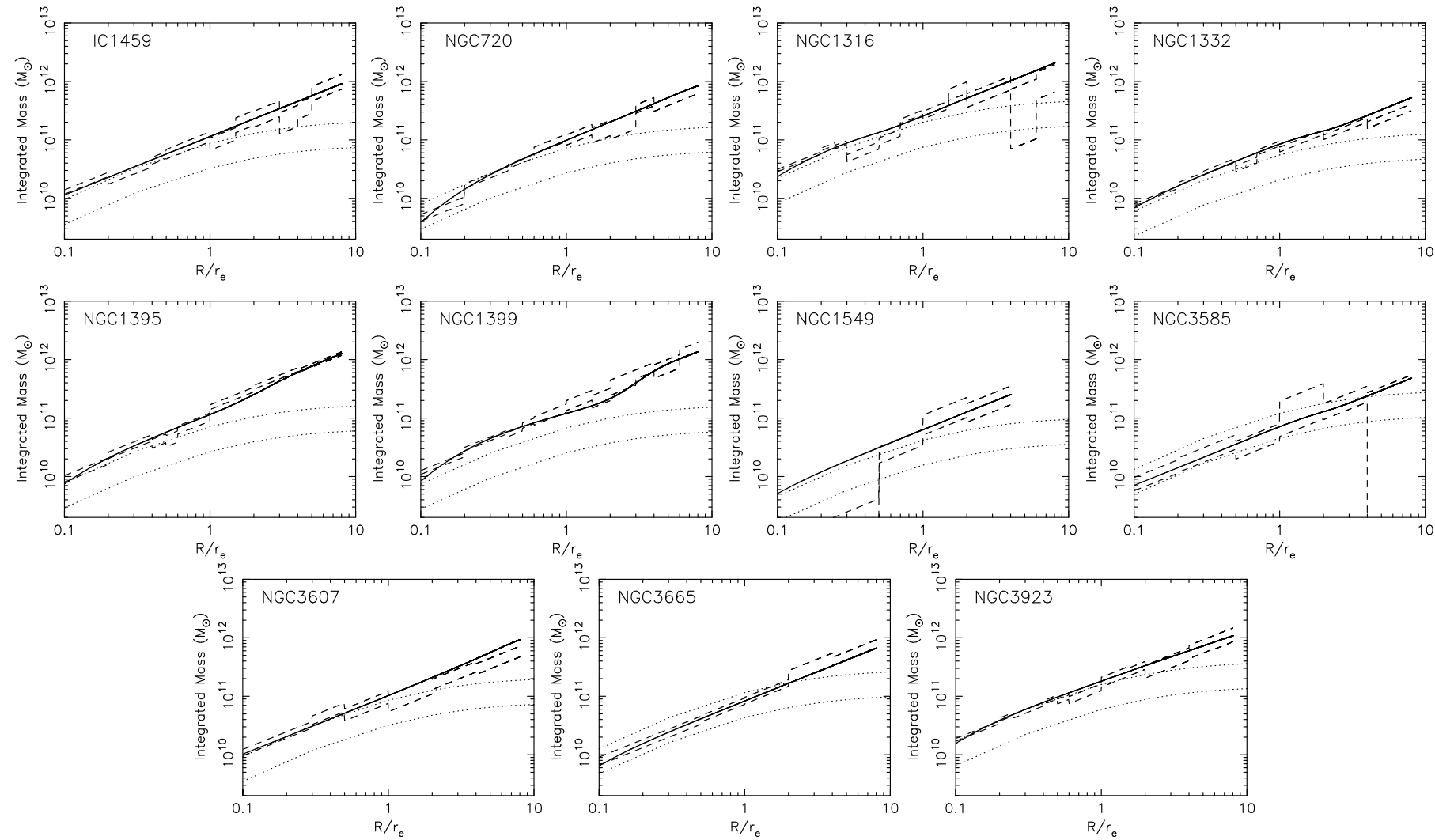

Fig. 8. Integrated mass profiles of galaxies. The solid lines are total gravitational mass obtained from the best-fit functions of temperature and ISM density profiles. The dashed lines correspond to the upper and lower limits derived from the local gradients of temperature and density. We also plotted the stellar mass profiles assuming stellar $M / L_{B}$ to be 3 and 8 (dotted lines).

is consistent with the stellar mass. Further, the gradients of the gravitational mass are similar to those of the stellar mass. In contrast, outside the radius of a few $r_{\mathrm{e}}$, the derived gravitational mass becomes much larger than the stellar mass. These results 

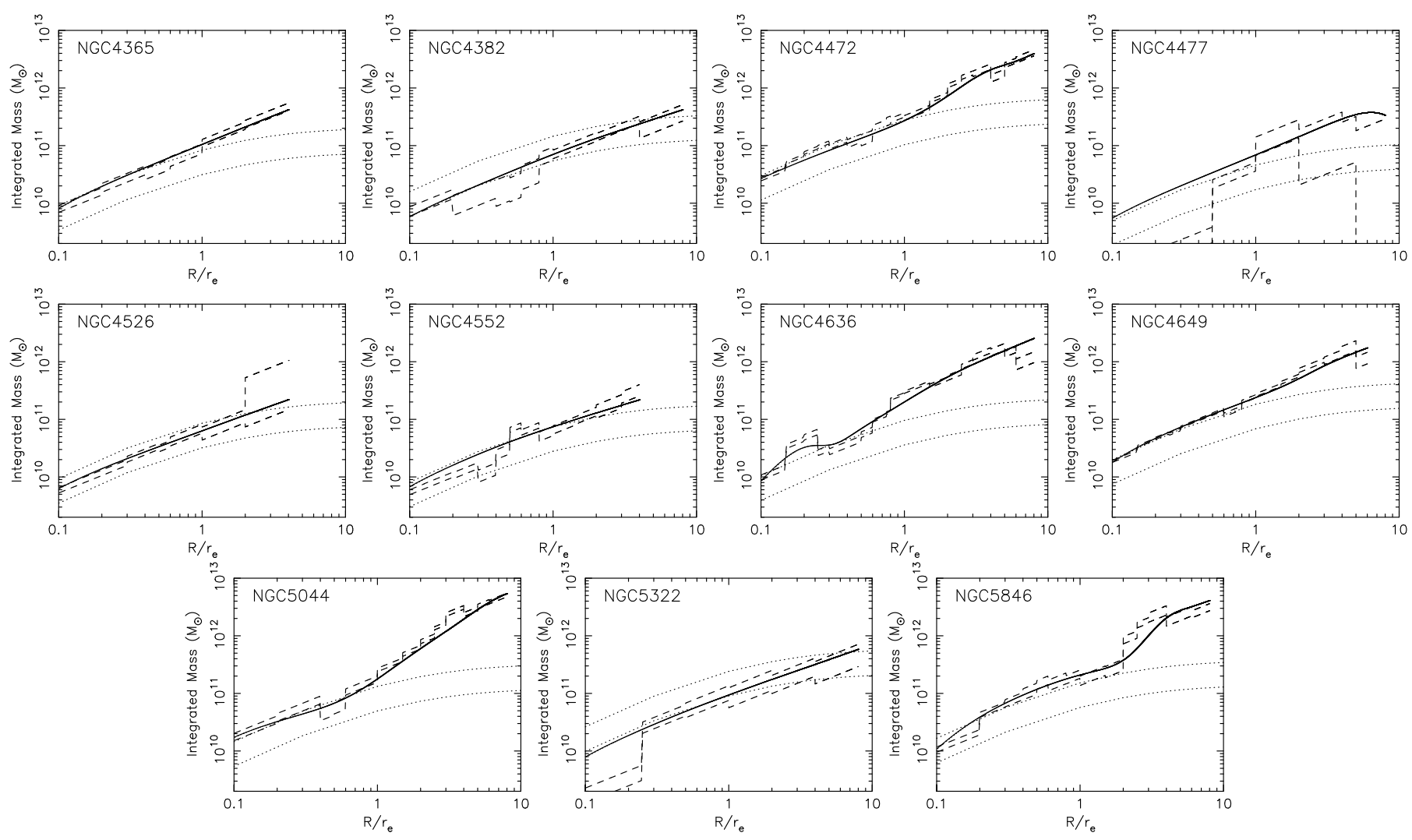

Fig. 8. continued.

indicate the existence of dark matter in the outer regions of earlytype galaxies.

When $L_{\mathrm{ISM}}$ is less than $80 \%$ of the total luminosity within $4 r_{\mathrm{e}}$, the total mass profile is calculated using the best-fit $\beta$-model of the density profile from the spectral fitting. The results are compared with those from the surface brightness in Fig. 18. The two methods give similar total mass profiles within 10-20\%. For NGC 3585 and NGC 5322, these discrepancies are $~ 30 \%$, but within the large errors of the mass profiles of the two galaxies. Thus, the mass profiles of these galaxies obtained from two methods are consistent within the error. Hereafter, we use the total mass profiles derived from the surface brightness profiles.

\section{Discussion}

The observed temperature profiles and X-ray luminosities of the ISM lead to a division of early-type galaxies into two categories: $X_{\mathrm{E}}$ galaxies and $X_{\mathrm{C}}$ galaxies. The $X_{\mathrm{E}}$ galaxies have increasing temperature profiles and $L_{\mathrm{ISM}} / L_{\sigma}>1$, whereas the $X_{\mathrm{C}}$ galaxies have flat or negative temperature gradients and $L_{\mathrm{ISM}} / L_{\sigma} \lesssim 1$. Here, $L_{\sigma}$ represents the expected energy input from stellar mass loss (Matsushita 2001). In Sect. 7.1, the derived ISM temperatures are compared with the stellar velocity dispersions. In Sects. 7.2 and 7.3, we derive gravitational mass-to-light ratios in the $B$ - and $K$-band, respectively, and constrain contributions from stellar mass and differences in dark mass between the $X_{\mathrm{C}}$ and $X_{\mathrm{E}}$ galaxies. Finally, in Sect. 7.4, we discuss dark matter distribution in early-type galaxies themselves and their luminosity.

\subsection{ISM temperature vs. stellar velocity dispersion}

Figure 9 shows the correlation between central ISM temperature and central stellar velocity dispersion $\sigma^{2}$ (Table 1).

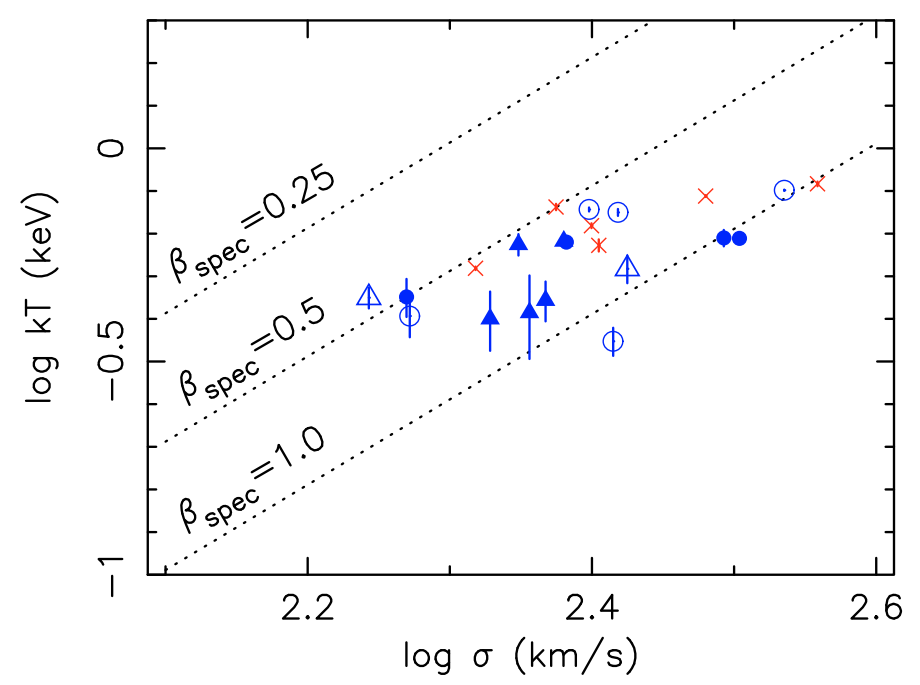

Fig. 9. Central ISM temperatures plotted against central stellar velocity dispersion, $\sigma$. Colors indicate galaxy categories for $X_{\mathrm{E}}$ galaxies (red crosses), field $X_{\mathrm{C}}$ galaxies (filled blue symbols), and $X_{\mathrm{C}}$ galaxies in clusters (open blue symbols). For $X_{\mathrm{C}}$ galaxies, the temperatures within $0.5 r_{\mathrm{e}}$ (circles) and $1 r_{\mathrm{e}}$ (triangles) are plotted for the data with high and poor statistics, respectively. The dotted lines correspond to $\beta_{\text {spec }}$ of $0.25,0.5$, and 1.0 from the top to bottom.

The temperature roughly correlates with the stellar velocity dispersion. The parameter $\beta_{\text {spec }}$ denotes the ratio of stellar velocity dispersion to ISM temperature with $\beta_{\text {spec }}=\mu m_{\mathrm{p}} \sigma^{2} / k T$, with $\mu$ indicating the mean molecular weight in terms of proton mass $m_{\mathrm{p}}$. For $X_{\mathrm{C}}$ galaxies, $\beta_{\mathrm{spec}}$ is about $\beta_{\text {spec }}=0.5-1.0$, which indicates that the ISM temperatures are consistent with heating due to stellar motion (Matsushita 2001). The galaxies with low $\sigma$ 
Table 6. Integrated $M / L_{B}$ and $M / L_{K}$ at $0.5,3$ and $6 r_{\mathrm{e}}$.

\begin{tabular}{|c|c|c|c|c|c|c|}
\hline Galaxy & $\begin{array}{c}M / L_{B}\left(<0.5 r_{\mathrm{e}}\right) \\
\left(M_{\odot} / L_{\odot}\right)\end{array}$ & $\begin{array}{c}M / L_{B}\left(<3 r_{\mathrm{e}}\right) \\
\left(M_{\odot} / L_{\odot}\right)\end{array}$ & $\begin{array}{c}M / L_{B}\left(<6 r_{\mathrm{e}}\right) \\
\left(M_{\odot} / L_{\odot}\right) \\
\end{array}$ & $\begin{array}{c}M / L_{K}\left(<0.5 r_{\mathrm{e}}\right) \\
\left(M_{\odot} / L_{\odot}\right)\end{array}$ & $\begin{array}{c}M / L_{K}\left(<3 r_{\mathrm{e}}\right) \\
\left(M_{\odot} / L_{\odot}\right)\end{array}$ & $\begin{array}{c}M / L_{K}\left(<6 r_{\mathrm{e}}\right) \\
\left(M_{\odot} / L_{\odot}\right)\end{array}$ \\
\hline IC 1459 & 8.8 & 18.1 & 29.9 & 1.4 & 2.9 & 4.8 \\
\hline NGC 720 & 8.7 & 19.5 & 33.6 & 1.7 & 3.9 & 6.7 \\
\hline NGC 1316 & 9.1 & 17.8 & 29.5 & 1.5 & 3.0 & 4.9 \\
\hline NGC 1332 & 10.6 & 16.5 & 27.3 & 1.7 & 2.7 & 4.4 \\
\hline NGC 1395 & 10.9 & 27.7 & 50.9 & 1.5 & 3.9 & 7.2 \\
\hline NGC 1399 & 14.3 & 27.1 & 58.1 & 1.6 & 3.0 & 6.4 \\
\hline NGC 1549 & 10.2 & 20.9 & - & 1.7 & 3.5 & - \\
\hline NGC 3585 & 4.0 & 6.9 & 11.4 & 0.7 & 1.2 & 1.9 \\
\hline NGC 3607 & 8.1 & 17.3 & 30.8 & 1.5 & 3.2 & 5.8 \\
\hline NGC 3665 & 4.8 & 10.0 & 16.6 & 0.9 & 1.8 & 3.0 \\
\hline NGC 3923 & 8.2 & 13.7 & 20.4 & 1.1 & 1.8 & 2.6 \\
\hline NGC 4365 & 8.4 & 17.4 & - & 1.6 & 3.2 & - \\
\hline NGC 4382 & 3.3 & 6.0 & 8.7 & 0.7 & 1.2 & 1.8 \\
\hline NGC 4472 & 6.5 & 23.8 & 40.4 & 1.3 & 4.7 & 7.9 \\
\hline NGC 4477 & 10.0 & 22.0 & 31.0 & 2.0 & 4.3 & 6.1 \\
\hline NGC 4526 & 5.3 & 9.2 & - & 0.9 & 1.5 & - \\
\hline NGC 4552 & 7.4 & 10.9 & - & 1.3 & 1.9 & - \\
\hline NGC 4636 & 9.8 & 43.0 & 75.3 & 1.7 & 7.3 & 12.8 \\
\hline NGC 4649 & 9.1 & 21.4 & 36.4 & 1.6 & 3.8 & 6.5 \\
\hline NGC 5044 & 6.9 & 41.5 & 109.9 & 1.0 & 6.0 & 15.9 \\
\hline NGC 5322 & 2.7 & 4.8 & 7.2 & 0.7 & 1.2 & 1.8 \\
\hline NGC 5846 & 10.5 & 30.8 & 80.6 & 1.6 & 4.8 & 12.5 \\
\hline
\end{tabular}

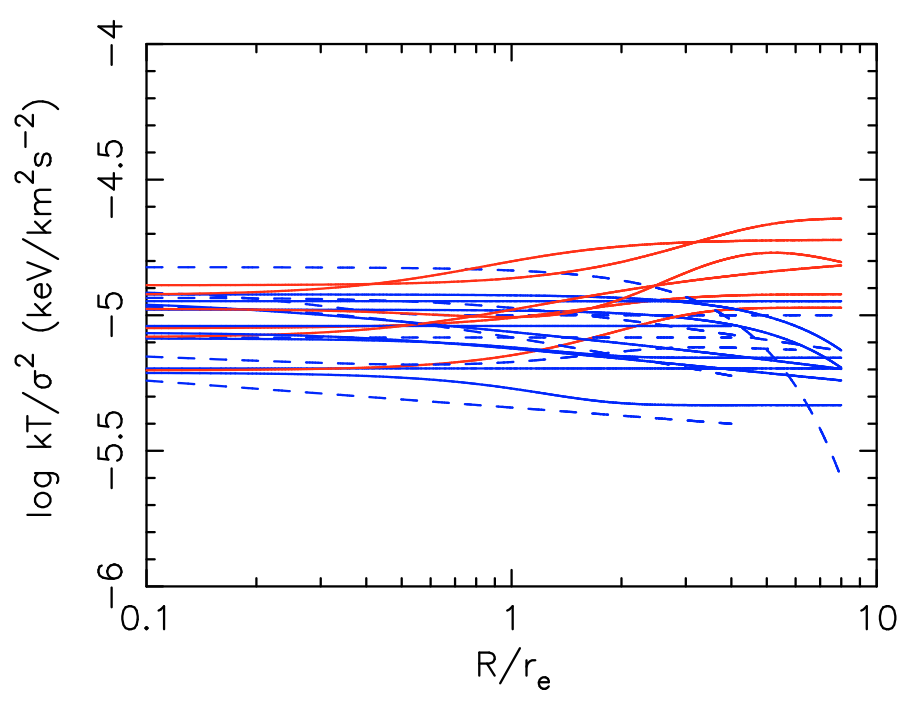

Fig. 10. Temperature profiles of the ISM scaled with central stellar velocity dispersion. The solid red, solid blue, and dashed blue lines represent the $X_{\mathrm{E}}$ galaxies, field $X_{\mathrm{C}}$ galaxies, and $X_{\mathrm{C}}$ galaxies in clusters, respectively.

tend to have low $\beta$ values. This may be due to a selection effect, since brighter galaxies with higher ISM temperatures were the first proposed for observation.

In Fig. 10, we plotted the temperature profiles of the sample galaxies scaled with central stellar velocity dispersion. There is no significant difference in the ISM temperature between the $X_{\mathrm{C}}$ and $X_{\mathrm{E}}$ galaxies at the central regions. Therefore, the ISM temperatures of the $X_{\mathrm{C}}$ and $X_{\mathrm{E}}$ galaxies would reflect the same potential. However, in the outer regions the $X_{\mathrm{E}}$ galaxies have higher ISM temperatures than the $X_{\mathrm{C}}$ galaxies for the same stellar velocity dispersion. This is due to the difference in potential due to the hot intra-group medium around the $X_{\mathrm{E}}$ galaxies.

\subsection{Mass-to-light ratio in $B$-band}

In order to compare the difference in dark matter profiles between the $X_{\mathrm{C}}$ and $X_{\mathrm{E}}$ galaxies, we used the mass-to-light ratio $\left(M / L_{B}\right)$. We assumed the de Vaucouleurs law for the stellar distribution. Figure 11 summarizes the integrated $M / L_{B}$ profiles. For NGC 1549, NGC 4477, and NGC 5322, profiles within $0.5 r_{\mathrm{e}}$ were not plotted in the figure, since we used only XMM-Newton data for those galaxies. From $0.2 r_{\mathrm{e}}$ to $1 r_{\mathrm{e}}, M / L_{B}$ of each $X_{\mathrm{C}}$ galaxy is nearly constant at $3-10$, which is consistent with typical values of stellar $M / L_{B}$. Even in the $X_{\mathrm{E}}$ galaxies, $M / L_{B}$ values within $0.5-1 r_{\mathrm{e}}$ are also flat. These results suggest that stellar mass dominates within $R<0.5-1 r_{\mathrm{e}}$. In NGC 4636, a wavy $M / L_{B}$ profile is seen at $R<0.3 r_{\mathrm{e}}$, which is artificially caused by the complicated X-ray structures in the central regions discovered by Chandra (Jones et al. 2002). Hereafter, we denote integrated $M / L_{B}$ within $s r_{\mathrm{e}}$ as $M / L_{B}\left(<s r_{\mathrm{e}}\right)$, where $s$ is a constant.

The top left panel of Fig. 12 shows the relationship of $M / L_{B}\left(<0.5 r_{\mathrm{e}}\right)$ to total $B$-band luminosity, $L_{B}$. In this plot, we add $M / L_{B}$ of the cD galaxy of the Virgo cluster, M 87, obtained from XMM-Newton observations (Matsushita et al. 2002). $M / L_{B}\left(<0.5 r_{\mathrm{e}}\right)$ of the $X_{\mathrm{E}}$ and $X_{\mathrm{C}}$ galaxies is about 10 , with significant scatter. A correlation between $M / L_{B}$ and $L_{B}$ elliptical galaxies was found by optical measurements (Gerhard et al. 2001), where gravitational mass was derived from stellar velocity dispersion in the central region of elliptical galaxies. Our $M / L_{B}$ and $L_{B}$ relation is scattered around the relation found by Gerhard et al. (2001).

On the other hand, at $R>1 r_{\mathrm{e}}$, the derived $M / L_{B}$ starts to increase (Fig. 11). The $X_{\mathrm{C}}$ galaxies have similarly shaped $M / L_{B}$ profiles. $M / L_{B}\left(<3 r_{\mathrm{e}}\right)$ and $M / L_{B}\left(<6 r_{\mathrm{e}}\right)$ of the $X_{\mathrm{C}}$ galaxies are about 6-25 $M_{\odot} / L_{\odot}$. The $X_{\mathrm{E}}$ galaxies have systematically higher $M / L_{B}$ values than the $X_{\mathrm{C}}$ galaxies at $>3 r_{\mathrm{e}}$. (Fig. 12). The $X_{\mathrm{E}}$ galaxies have $M / L_{B}\left(<3 r_{\mathrm{e}}\right)$ and $M / L_{B}\left(<6 r_{\mathrm{e}}\right)$ of $25-40 M_{\odot} / L_{\odot}$ and 40-100 $M_{\odot} / L_{\odot}$, respectively. These results indicate that dark 

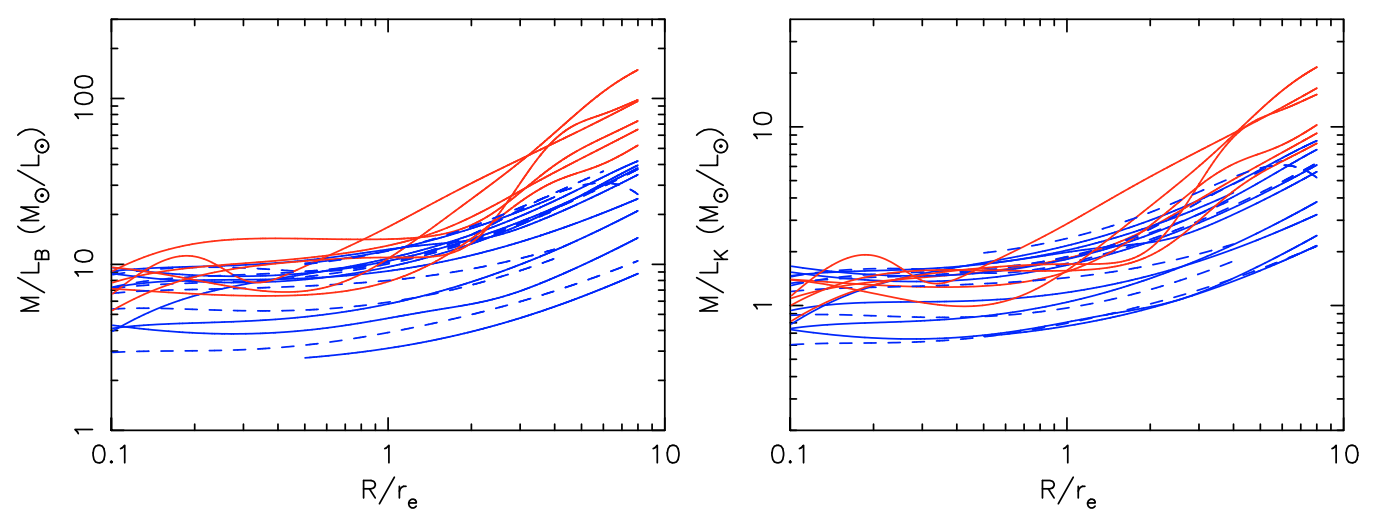

Fig. 11. Profiles of integrated $M / L_{B}$ (left) and $M / L_{K}$ (right). The meanings of colors and lines are the same as those in Fig. 10.
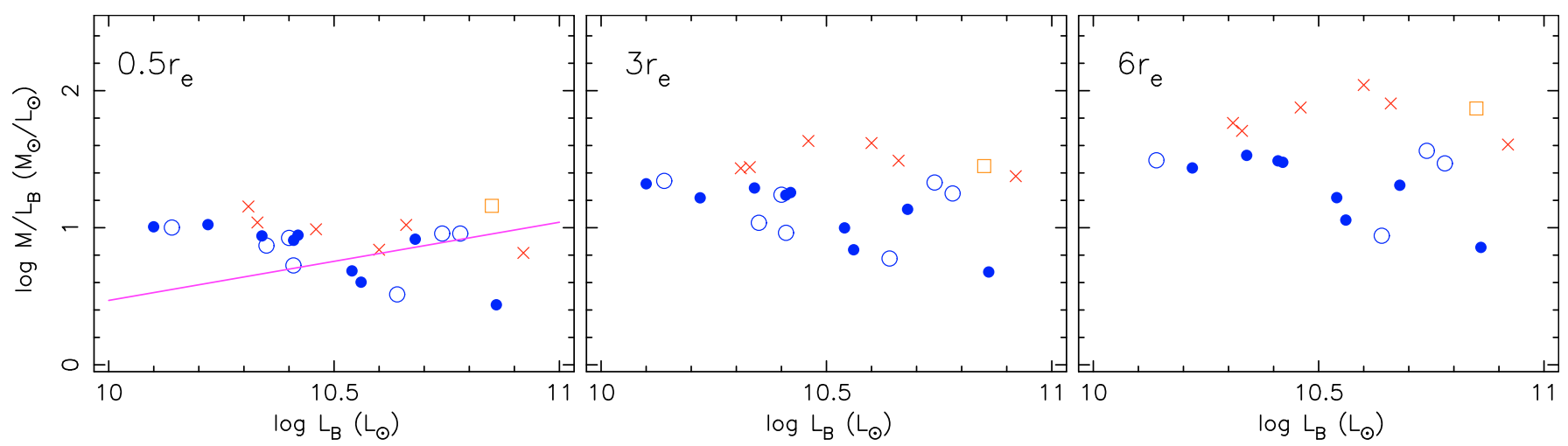

Fig. 12. $M / L_{B}\left(<0.5 r_{\mathrm{e}}\right)($ left $), M / L_{B}\left(<3 r_{\mathrm{e}}\right)($ center $)$, and $M / L_{B}\left(<6 r_{\mathrm{e}}\right)(r i g h t)$ against $L_{B}$. The quadrangle represents the value of M87 by Matsushita et al. (2002). Meanings of other symbols are the same as those in Fig. 3. The solid line represents the correlation derived from the stellar velocity dispersion by Gerhard et al. (2001).

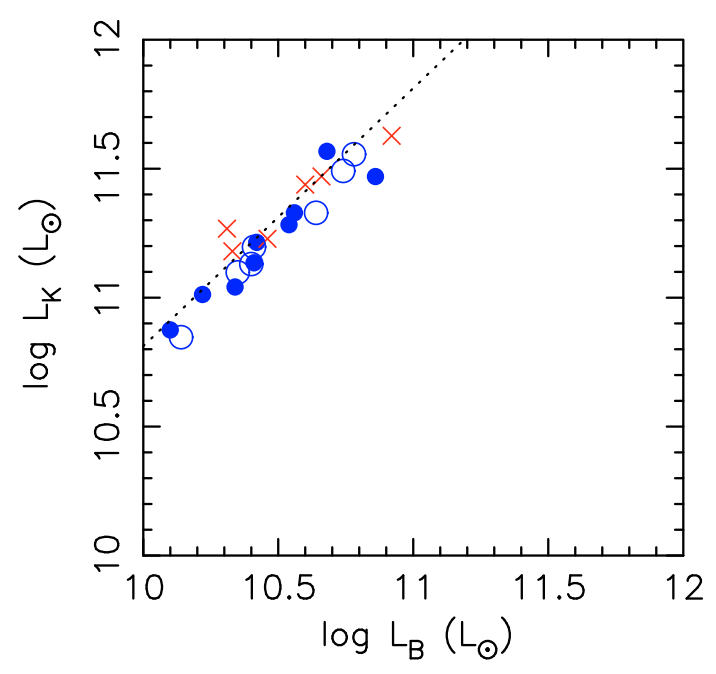

Fig. 13. Relationship between $B$-band and $K$-band luminosity of the sample galaxies. Meanings of the symbols are the same as those in Fig. 3. The dotted line corresponds to the appropriate color $B-K=4.2$ for early-type galaxies (Lin \& Mohr 2004).

matter is common in early-type galaxies. In addition, the $X_{\mathrm{E}}$ galaxies have more dark matter than the $X_{\mathrm{C}}$ galaxies.

\subsection{Mass-to-light ratios in $\mathrm{K}$-band}

Historically, the $B$-band mass-to-light ratio has been used in such studies. However, $K$-band luminosity well describes the stellar mass. Thus, we also derived the $K$-band mass-to-light ratio to study the dark matter profiles. We calculated the $K$-band luminosity $L_{K}$ from the Two Micron All Sky Survey (2MASS). The effect of Galactic extinction was corrected using the NASA/IPAC Extragalactic Database (NED). The relationship between $L_{B}$ and $L_{K}$ is close to that of $B-K=4.2$, which is the appropriate color of stars in early-type galaxies (Lin \& Mohr 2004), with some scatter (Fig. 13).

The right panel of Fig. 11 shows the integrated profiles of the $K$-band mass-to-light ratio, $M / L_{K}$, of the sample galaxies. At the central region, the $X_{\mathrm{E}}$ and $X_{\mathrm{C}}$ galaxies have similar $M / L_{K}$ at $\sim 1$.

In Fig. 14, we compared the relationship of $M / L_{K}\left(<0.5 r_{\mathrm{e}}\right)$, $M / L_{K}\left(<3 r_{\mathrm{e}}\right)$, and $M / L_{K}\left(<6 r_{\mathrm{e}}\right)$ with $L_{K}$ except M 87. For earlytype galaxies, the stellar $K$-band mass-to-light ratio, $M / L_{K}$, is about $1 M_{\odot} / L_{\odot}$. The scatter of $M / L_{K}\left(<0.5 r_{\mathrm{e}}\right)$ values becomes smaller than that of $M / L_{B}\left(<0.5 r_{\mathrm{e}}\right)$ values (Fig. 14). Both the $X_{\mathrm{E}}$ and $X_{\mathrm{C}}$ galaxies have $M / L_{K}$ values of $\sim 1 M_{\odot} / L_{\odot}$, and no correlation with $L_{K}$. This result indicates that stars dominate the mass within $0.5 r_{\mathrm{e}}$, and we observed the stellar $M / L_{K}$ in these region, except the cD galaxy M 87. M 87 may contain a similar amount of dark matter with stellar mass within $0.5 r_{\mathrm{e}}$, since $M / L_{K}$ is a factor of 2 larger than those of $X_{\mathrm{E}}$ and $X_{\mathrm{C}}$ galaxies. On the other hand, the $M / L_{K}\left(<3 r_{\mathrm{e}}\right)$ values of the $X_{\mathrm{E}}$ and $X_{\mathrm{C}}$ galaxies are 4-10 and 1-4 $M_{\odot} / L_{\odot}$, respectively (Fig. 14). The $M / L_{K}\left(<6 r_{\mathrm{e}}\right)$ values of the $X_{\mathrm{E}}$ and $X_{\mathrm{C}}$ galaxies are $\sim 10$ and $2-6 M_{\odot} / L_{\odot}$, respectively (Fig. 14). 

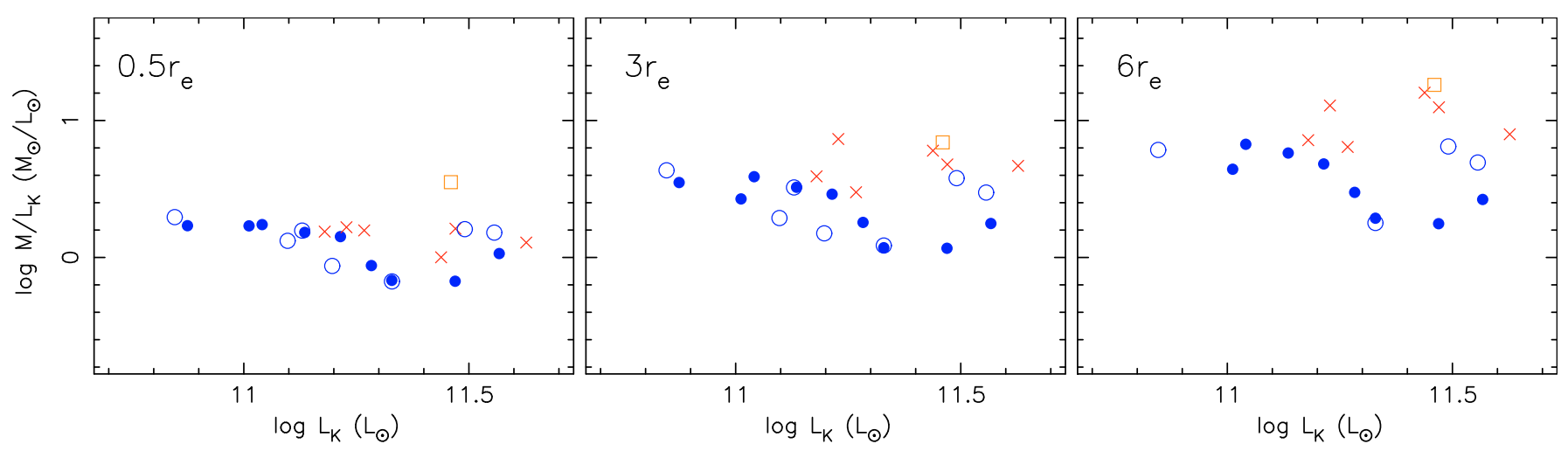

Fig. 14. Integrated $M / L_{K}\left(<0.5 r_{\mathrm{e}}\right)($ left $), M / L_{K}\left(<3 r_{\mathrm{e}}\right)($ center $)$, and $M / L_{K}\left(<6 r_{\mathrm{e}}\right)($ right $)$ against $L_{K}$. Meanings of the symbols are the same as those in Fig. 12.

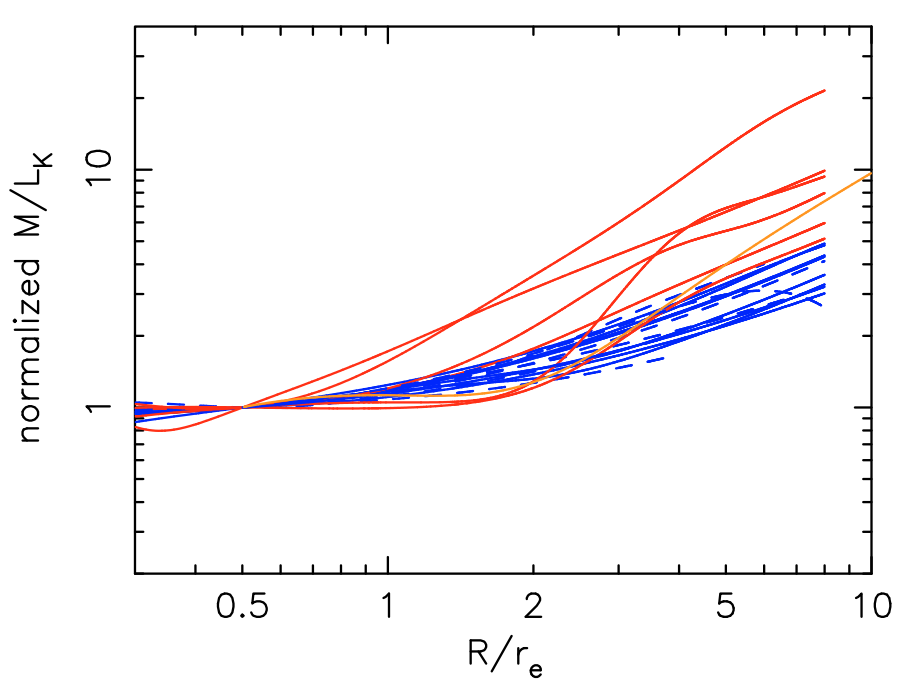

Fig. 15. Profiles of $M / L_{K}$ normalized by $M / L_{K}\left(<0.5 r_{\mathrm{e}}\right)$. We also plotted the $M / L_{K}$ profile of M 87 by Matsushita et al. (2002) (orange solid line). The meanings of other colors and lines are the same as those in Fig. 10.

\subsection{Dark matter in early-type galaxies}

We normalized the $M / L_{K}$ profile with $M / L_{K}\left(<0.5 \quad r_{\mathrm{e}}\right)$ for each galaxy (Fig. 15). Then, the profiles of mass-to-light ratios of $X_{\mathrm{C}}$ galaxies have similarities. We also derived the ratio of $M / L_{K}\left(<3 r_{\mathrm{e}}\right)$ and $M / L_{K}\left(<6 r_{\mathrm{e}}\right)$ to $M / L_{K}\left(<0.5 r_{\mathrm{e}}\right)$ for each galaxy. The ratios of $M / L_{K}\left(<3 r_{\mathrm{e}}\right)$ and $M / L_{K}\left(<6 r_{\mathrm{e}}\right)$ to $M / L_{K}\left(<0.5 r_{\mathrm{e}}\right)$ are similar among the $X_{\mathrm{C}}$ galaxies at 2 and $3-4$, respectively (Fig. 16). Considering that stellar mass dominates within $1 r_{\mathrm{e}}$, and assuming that the stellar $M / L_{K}$ is nearly constant within a galaxy, the $X_{\mathrm{C}}$ galaxies contain similar amounts of dark matter. Within $3 r_{\mathrm{e}}$, the mass of dark matter is similar to the stellar mass, while within $6 r_{\mathrm{e}}$, the former is $2-3$ times larger than the latter. These ratios should reflect the potential of early-type galaxies themselves. Thus, the dark mass distribution in early-type galaxies is slightly more extended than that of stars. It is thought that early-type galaxies have 10 times more dark mass than stellar mass as in spiral galaxies (e.g., Ciotti et al. 1991). However, the galaxies themselves may not contain such large amounts of mass, at least within several times $r_{\mathrm{e}}$. This result is important for the study of the origin of the dark matter content in early-type galaxies and for the study of the formation and evolution of these galaxies.

In contrast, the $X_{\mathrm{E}}$ galaxies have systematically larger ratios; i.e., they have more dark matter in their outer regions. Figure 17 shows that the galaxies with a larger $L_{\mathrm{ISM}} / L_{B} \sigma^{2}$ have more dark matter at $R>1 r_{\mathrm{e}}$. The ratio of $\mathrm{M} 87$ may also lie in the relation between $L_{\mathrm{ISM}} / L_{B} \sigma^{2}$ and dark to stellar mass ratio, considering that M 87 may contain a similar amount of dark mass to stellar mass within $0.5 r_{\mathrm{e}}$. In other words, the X-ray luminosity of the $X_{\mathrm{E}}$ galaxies may be determined in relation to their potential structure, as indicated by Matsushita (2001) and Matsushita et al. (2002). The difference in temperature profiles between the $X_{\mathrm{E}}$ and $X_{\mathrm{C}}$ galaxies would be due to differences in the surrounding gravitational potential. These results suggest that the $\mathrm{X}$-ray luminous early-type galaxies commonly sit in the center of a large-scale (a few hundred kpc) potential well, which leads to their high luminosities. Other galaxies may lack such a largescale potential well and contain their own dark matter.

\section{Conclusion}

We analyzed 22 early-type galaxies using XMM-Newton and Chandra data. To derive the gravitational mass profiles, we obtained the temperature and ISM density profiles through spectral fitting and spatial analysis, respectively. We classified the galaxies into two categories, $X_{\mathrm{E}}$ and $X_{\mathrm{C}}$ galaxies, on the basis of whether the temperature gradient is positive or negative toward the outer radius. The ISM luminosity of the $X_{\mathrm{C}}$ galaxies is consistent with the energy input from stellar mass loss. In contrast, the $X_{\mathrm{E}}$ galaxies have higher ISM luminosity.

At the central regions, $R<0.5-1 r_{\mathrm{e}}$, the derived integrated $M / L_{K}$ of both of the $X_{\mathrm{C}}$ and $X_{\mathrm{E}}$ galaxies are about 1 and have smaller scatter than $M / L_{B}$. The values and profiles of $M / L_{K}$ indicate that stellar mass dominates the total mass in these regions. In the outer regions, $M / L_{B}$ and $M / L_{K}$ of the $X_{\mathrm{E}}$ galaxies are higher than those of the $X_{\mathrm{C}}$ galaxies.

On the basis of these results, we can conclude the following. The normal early-type galaxies, $X_{\mathrm{C}}$ galaxies, contain their own dark matter at amounts that are 1.5-2 times larger than the stellar mass within $5 r_{\mathrm{e}}$. The $X_{\mathrm{E}}$ galaxies are located as the central galaxy in a larger scale potential structure, such as a galaxy group. This fact directly affects the gravitational potential profile of the galaxy itself, and causes it to contain significantly higher amounts of dark matter than that in the $X_{\mathrm{C}}$ galaxies. This 

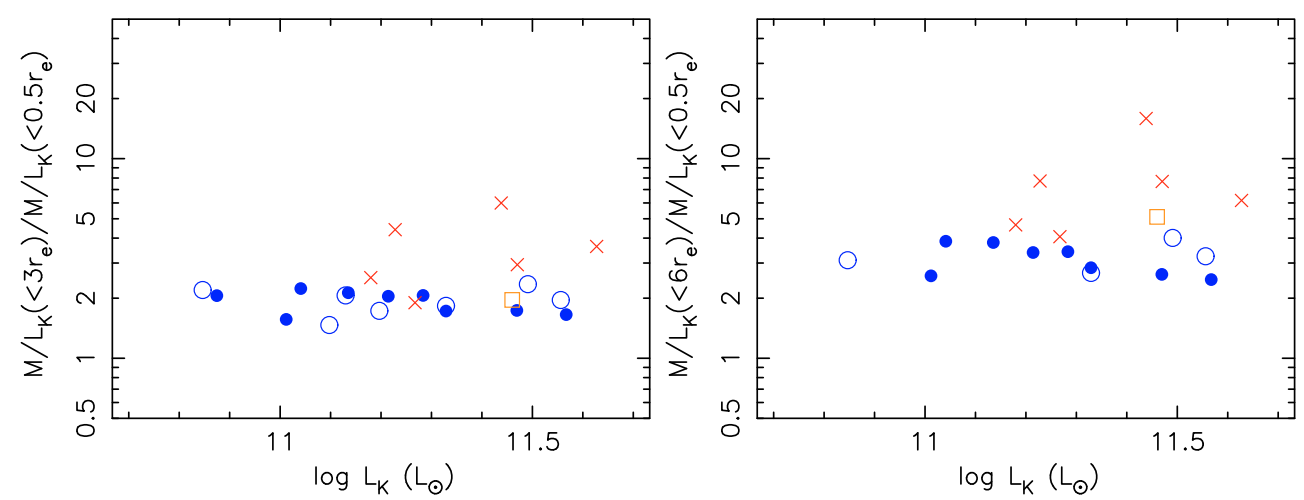

Fig. 16. Ratio of the integrated $M / L_{K}\left(<3 r_{\mathrm{e}}\right)$ and $M / L_{K}\left(<6 r_{\mathrm{e}}\right)$ to $M / L_{K}\left(<0.5 r_{\mathrm{e}}\right)$ plotted against $L_{K}$. Meanings of the symbols are the same as those in Fig. 12.
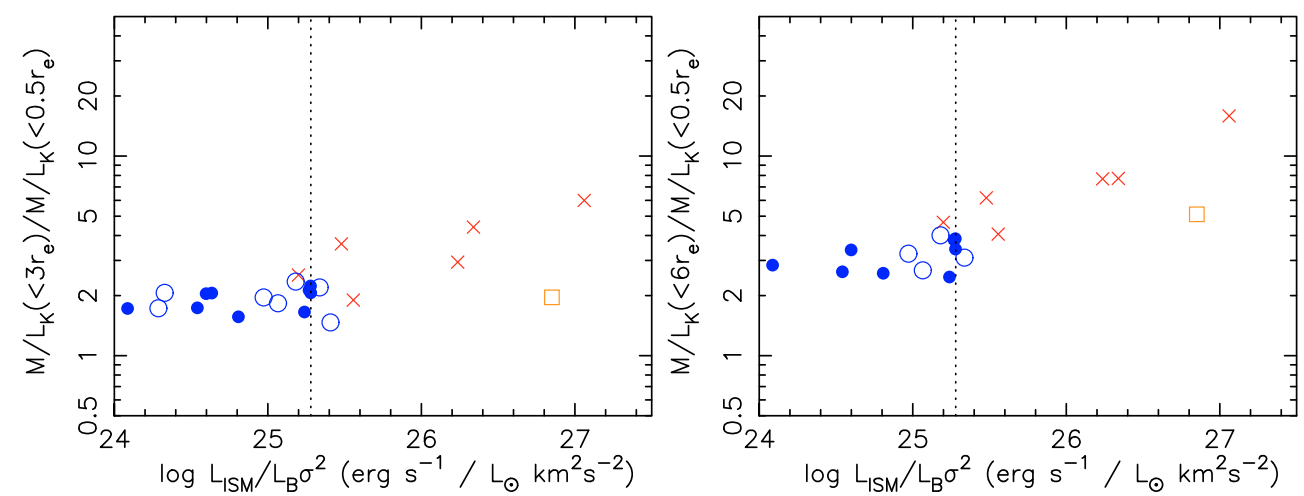

Fig. 17. Ratio of the integrated $M / L_{K}\left(<3 r_{\mathrm{e}}\right)$ and $M / L_{K}\left(<6 r_{\mathrm{e}}\right)$ to $M / L_{K}\left(<0.5 r_{\mathrm{e}}\right)$ plotted against $L_{\mathrm{ISM}} / L_{B} \sigma^{2}$. Meanings of the symbols are the same as those in Fig. 12. The dotted line represents the kinetic heating rate by stellar mass loss.

difference in the gravitational potential leads to the difference in the temperature profile and X-ray ISM luminosity between the $X_{\mathrm{E}}$ and $X_{\mathrm{C}}$ galaxies.

\section{References}

Anders, E., \& Grevesse, N. 1989, Geochim. Cosmochim. Acta, 53, 197 Athey, A. E 2007, [arXiv: 0711.0395]

Bender, R., Burstein, D., \& Faber, S. M. 1993, ApJ, 411, 153

Beuing, J., Dobereiner, S., Bohringer, H., \& Bender, R. 1999, MNRAS, 302, 209

Blanton, E. L., Sarazin, C. L., \& Irwin, J. A. 2001, ApJ, 552, 106

Chakrabarty, D., \& Raychaudhury, S. 2008, AJ, 135, 2350

Canizares, C. R., Fabbiano, G., \& Trinchieri, G. 1987, ApJ, 312, 503

Ciotti, L., D'Ercole, A., Pellegrini, S., \& Renzini, A. 1991, ApJ, 376, 380

Dickey, J. M., \& Lockman, F. J. 1990, ARA\&A, 28, 215

Diehl, S., \& Statler, T. S. 2007, ApJ, 668, 150

Diehl, S., \& Statler, T. S. 2008, ApJ, 680, 897

Djorgovski, S., \& Davis, M. 1987, ApJ, 313, 59

Fabbiano, G. Elvis, M., Markoff, S., et al. 2003, ApJ, 588, 175

Forman, W., Jones, C., \& Tucker, W. 1985, ApJ, 293, 102

Fukazawa, Y., Botoya-Nonesa, J. G., Pu, J., Ohto, A., \& Kawano, N. 2006, ApJ, 636, 698

Gerhard, O., Kronawitter, A., Saglia, R. P., \& Bender, R. 2001, AJ, 121, 1936

Humphrey, P. J., Buote, D. A., Gastaldello, F., et al. 2006, ApJ, 646, 899

Jones, C., Forman, W., Vikhlinin, A., et al. 2002, ApJ, 567, L115

Kronawitter, A., Saglia, R. P., Gerhard, O., \& Bender, R. 2000, A\&AS, 144, 53

Lin, Y.-T., \& Mohr, J. J. 2004, ApJ, 617, 879

Machacek, M., Jones, C., Forman, W. R., \& Nulsen, P. 2006, ApJ, 644, 155
Mathews, W. G., Brighenti, F., Faltenbacher, A., et al. 2006, ApJ, 652, L17 Matsushita, K. 2001, ApJ, 547, 693

Matsushita, K., Makishima, K., Awaki, H., et al. 1994, ApJ, 436, L41

Matsushita, K., Makishima, K., Ikebe, Y., et al. 1998, ApJ, 499, L13

Matsushita, K., Ohashi, T., \& Makishima, K. 2000, PASJ, 52, 685

Matsushita, K., Belsole, E., Finoguenov, A., \& Boehringer, H. 2002, A\&A, 386, 77

Matsushita, K., Fukazawa, Y., Hughes, J. P., et al. 2007, PASJ, 59, 327

Mellier, Y., \& Mathez, G. 1987, A\&A, 175, 1

Navarro, J. F., Frenk, C. S., \& White, S. D. M. 1996, ApJ, 462, 563

Navarro, J. F., Frenk, C. S., \& White, S. D. M. 1997, ApJ, 490, 493

O'Sullivan, E., Ponman, T. J., \& Collins, R. S. 2003, MNRAS, 340, 1375

Prugniel, P., \& Simien, F. 1996, A\&A, 309, 749

Randall, S. W., Sarazin, C. L., \& Irwin, J. A. 2004, ApJ, 600, 729

Romanowsky, A. J., Douglas, N. G., Arnaboldi, M., et al. 2003, Science, 301, 1696

Rubin, V. C., Thonnard, N., \& Ford, W. K., Jr. 1978, ApJ, 225, L107

Smith, R. K., Brickhouse, N. S., Liedahl, D. A., \& Raymond, J. C. 2001, ApJ, 556, L91

Takahashi, I. 2004, Ph.D. Thesis, Univ. of Tokyo

Tawara, Y., Matsumoto, C., Tozuka, M., et al. 2008, PASJ, 60, 307

Tully, R. B. 1988, Nearby Galaxy Catalog (Cambridge and New York: Cambridge University Press), 221

Xu, H., Kahn, S. M., Peterson, J. R., et al. 2002, ApJ, 579, 600

Zappacosta, L., Buote, D. A., Gastaldello, F., et al. 2006, ApJ, 650, 777

de Vaucouleurs, G., de Vaucouleurs, A., Corwin, H. G., Jr., et al. 1991, Third Reference Catalogue of Bright Galaxies, RC3 Catalog

van Albada, T. S., Bahcall, J. N., Begeman, K., \& Sancisi, R. 1985, ApJ, 295, 305 
R. Nagino and K. Matsushita: Gravitational potential and X-ray luminosities of early-type galaxies, Online Material p 1

Table 3. Spectral fitting results of the projected annular spectra.

\begin{tabular}{|c|c|c|c|c|c|c|c|c|}
\hline Galaxy & $\begin{array}{c}\operatorname{Ring}^{a} \\
\left(r_{\mathrm{e}}\right)\end{array}$ & $\begin{array}{c}k T^{b} \\
(\mathrm{keV})\end{array}$ & $\begin{array}{c}\mathrm{O} \\
\text { (solar) } \\
\end{array}$ & $\begin{array}{c}\mathrm{Si} \\
\text { (solar) }\end{array}$ & $\begin{array}{c}\mathrm{Fe} \\
\text { (solar) }\end{array}$ & $\begin{array}{c}\log L_{\mathrm{ISM}}{ }^{b} \\
(\mathrm{erg} / \mathrm{s})\end{array}$ & $\begin{array}{c}\log L_{\mathrm{hard}}{ }^{b} \\
(\mathrm{erg} / \mathrm{s})\end{array}$ & $\chi^{2} /$ d.o.f. \\
\hline IC 1459 & $\begin{array}{c}0-0.5 \\
0.5-1 \\
1-2 \\
2-4 \\
4-8 \\
\end{array}$ & $\begin{array}{l}0.606_{-0.022}^{+0.022} \\
0.622_{-0.026}^{+0.026} \\
0.574_{-0.022}^{+0.021} \\
0.589_{-0.023}^{+0.022} \\
0.638_{-0.022}^{+0.028} \\
\end{array}$ & $\begin{array}{c}0.56_{-0.14}^{+0.18} \\
- \\
- \\
- \\
-\end{array}$ & $\begin{array}{c}0.54_{-0.38}^{+0.42} \\
- \\
- \\
- \\
-\end{array}$ & $\begin{array}{c}0.36_{-0.06}^{+0.03} \\
- \\
- \\
- \\
-\end{array}$ & $\begin{array}{l}39.60 \\
39.32 \\
39.47 \\
39.48 \\
39.68 \\
\end{array}$ & $\begin{array}{l}40.07 \\
39.54 \\
39.42 \\
39.12 \\
38.99 \\
\end{array}$ & $\begin{array}{c}242 / 201 \\
- \\
- \\
-\end{array}$ \\
\hline NGC 720 & $\begin{array}{l}0-1 \\
1-2 \\
2-4 \\
4-8 \\
\end{array}$ & $\begin{array}{l}0.601_{-0.010}^{+0.010} \\
0.564_{-0.012}^{+0.013} \\
0.554_{-0.012}^{+0.013} \\
0.480_{-0.034}^{+0.038} \\
\end{array}$ & $\begin{array}{c}0.49_{-0.08}^{+0.07} \\
- \\
- \\
-\end{array}$ & $\begin{array}{c}0.31_{-0.26}^{+0.24} \\
- \\
- \\
-\end{array}$ & $\begin{array}{c}0.50_{-0.03}^{+0.03} \\
- \\
- \\
-\end{array}$ & $\begin{array}{l}40.01 \\
39.93 \\
40.01 \\
39.94 \\
\end{array}$ & $\begin{array}{l}39.46 \\
39.23 \\
39.56 \\
39.54 \\
\end{array}$ & $\begin{array}{c}207 / 157 \\
- \\
-\end{array}$ \\
\hline NGC 1316 & $\begin{array}{c}0-0.25 \\
0.25-0.5 \\
0.5-1 \\
1-2 \\
2-4 \\
4-8 \\
\end{array}$ & $\begin{array}{l}0.728_{-0.005}^{+0.006} \\
0.632_{-0.007}^{+0.000} \\
0.599_{-0.006}^{+0.007} \\
0.664_{-0.009}^{+0.009} \\
0.373_{-0.013}^{+0.013} \\
0.616_{-0.046}^{+0.066}\end{array}$ & $\begin{array}{c}0.48_{-0.03}^{+0.03} \\
- \\
- \\
- \\
-\end{array}$ & $\begin{array}{c}0.24_{-0.05}^{+0.04} \\
- \\
- \\
- \\
-\end{array}$ & $\begin{array}{c}0.36_{-0.01}^{+0.01} \\
- \\
- \\
- \\
-\end{array}$ & $\begin{array}{l}40.09 \\
39.82 \\
40.00 \\
39.87 \\
39.81 \\
39.90 \\
\end{array}$ & $\begin{array}{l}39.45 \\
39.31 \\
39.61 \\
39.35 \\
39.68 \\
39.77 \\
\end{array}$ & $\begin{array}{c}1602 / 939 \\
- \\
- \\
- \\
-\end{array}$ \\
\hline NGC 1332 & $\begin{array}{c}0-0.5 \\
0.5-1 \\
1-2 \\
2-4 \\
4-8\end{array}$ & $\begin{array}{l}0.617_{-0.007}^{+0.003} \\
0.580_{-0.011}^{+0.011} \\
0.486_{-0.014}^{+0.015} \\
0.495_{-0.015}^{+0.008} \\
0.542_{-0.020}^{+0.015}\end{array}$ & $\begin{array}{c}0.72_{-0.08}^{+0.09} \\
- \\
- \\
-\end{array}$ & $\begin{array}{c}0.89_{-0.18}^{+0.10} \\
- \\
- \\
-\end{array}$ & $\begin{array}{c}0.56_{-0.05}^{+0.05} \\
- \\
- \\
-\end{array}$ & $\begin{array}{l}39.72 \\
39.36 \\
39.40 \\
39.49 \\
39.34 \\
\end{array}$ & $\begin{array}{l}39.14 \\
39.06 \\
39.15 \\
39.18 \\
38.89\end{array}$ & $\begin{array}{c}337 \text { / } 227 \\
- \\
- \\
-\end{array}$ \\
\hline NGC 1395 & $\begin{array}{c}0-0.5 \\
0.5-1 \\
1-2 \\
2-4 \\
4-8 \\
\end{array}$ & $\begin{array}{l}0.597_{-0.010}^{+0.009} \\
0.607_{-0.009}^{+0.009} \\
0.710_{-0.010}^{+0.010} \\
0.748_{-0.010}^{+0.010} \\
0.762_{-0.021}^{+0.020} \\
\end{array}$ & $\begin{array}{c}0.79_{-0.10}^{+0.23} \\
- \\
- \\
-\end{array}$ & $\begin{array}{c}1.15_{-0.23}^{+0.27} \\
- \\
- \\
-\end{array}$ & $\begin{array}{c}0.80_{-0.02}^{+0.02} \\
- \\
- \\
-\end{array}$ & $\begin{array}{l}39.63 \\
39.67 \\
39.89 \\
40.02 \\
40.02 \\
\end{array}$ & $\begin{array}{l}39.34 \\
39.37 \\
39.69 \\
39.71 \\
39.94 \\
\end{array}$ & $\begin{array}{c}381 / 342 \\
- \\
- \\
-\end{array}$ \\
\hline NGC 1399 & $\begin{array}{c}0-0.5 \\
0.5-1 \\
1-2 \\
2-4 \\
4-6 \\
6-8 \\
\end{array}$ & $\begin{array}{l}0.846_{-0.007}^{+0.007} \\
0.987_{-0.013}^{+0.013} \\
1.169_{-0.022}^{+0.022} \\
1.342_{-0.011}^{+0.011} \\
1.440_{-0.043}^{+0.036} \\
1.535_{-0.043}^{+0.032}\end{array}$ & $\begin{array}{c}0.72_{-0.13}^{+0.14} \\
- \\
- \\
- \\
-\end{array}$ & $\begin{array}{c}1.24_{-0.14}^{+0.15} \\
- \\
- \\
- \\
-\end{array}$ & $\begin{array}{c}1.07_{-0.08}^{+0.09} \\
- \\
- \\
- \\
-\end{array}$ & $\begin{array}{l}40.52 \\
40.36 \\
40.41 \\
40.73 \\
40.62 \\
40.43 \\
\end{array}$ & $\begin{array}{l}39.56 \\
39.44 \\
39.93 \\
39.77 \\
39.85 \\
39.87 \\
\end{array}$ & $\begin{array}{c}374 \text { / } 250 \\
- \\
- \\
- \\
-\end{array}$ \\
\hline NGC 1549 & $\begin{array}{l}0-1 \\
1-4 \\
\end{array}$ & $\begin{array}{l}0.398_{-0.051}^{+0.075} \\
0.441_{-0.076}^{+0.088} \\
\end{array}$ & $\begin{array}{c}0.25_{-0.09}^{+0.13} \\
-\end{array}$ & $\begin{array}{c}1.00^{*} \\
- \\
\end{array}$ & $\begin{array}{c}0.27_{-0.09}^{+0.06} \\
-\end{array}$ & $\begin{array}{l}39.14 \\
39.03 \\
\end{array}$ & $\begin{array}{l}39.07 \\
37.73 \\
\end{array}$ & $\begin{array}{c}93 / 111 \\
-\end{array}$ \\
\hline NGC 3585 & $\begin{array}{l}0-1 \\
1-2 \\
2-4 \\
4-8 \\
\end{array}$ & $\begin{array}{l}0.412_{-0.076}^{+0.107} \\
0.382_{-0.089}^{+0.131} \\
0.338_{-0.039}^{+0.057} \\
0.399_{-0.067}^{+0.068} \\
\end{array}$ & $\begin{array}{c}0.78_{-0.49}^{+0.60} \\
- \\
- \\
-\end{array}$ & $\begin{array}{c}1.00^{*} \\
- \\
- \\
-\end{array}$ & $\begin{array}{c}1.03_{-0.74}^{+33.81} \\
- \\
- \\
-\end{array}$ & $\begin{array}{l}38.69 \\
38.70 \\
39.11 \\
39.41 \\
\end{array}$ & $\begin{array}{l}39.12 \\
39.00 \\
38.14 \\
39.06 \\
\end{array}$ & $\begin{array}{c}241 / 159 \\
- \\
-\end{array}$ \\
\hline NGC 3607 & $\begin{array}{l}0-1 \\
1-2 \\
2-4 \\
4-8\end{array}$ & $\begin{array}{l}0.595_{-0.034}^{+0.034} \\
0.550_{-0.050}^{+0.032} \\
0.566_{-0.022}^{+0.022} \\
0.449_{-0.021}^{+0.035} \\
\end{array}$ & $\begin{array}{c}0.09_{-0.06}^{+0.07} \\
- \\
- \\
-\end{array}$ & $\begin{array}{c}0.18_{-0.18}^{+0.45} \\
- \\
-\end{array}$ & $\begin{array}{c}0.24_{-0.04}^{+0.03} \\
- \\
- \\
-\end{array}$ & $\begin{array}{l}39.68 \\
39.73 \\
40.13 \\
40.20 \\
\end{array}$ & $\begin{array}{l}39.51 \\
39.26 \\
39.34 \\
40.18 \\
\end{array}$ & $\begin{array}{c}177 \text { / } 193 \\
- \\
- \\
-\end{array}$ \\
\hline NGC 3665 & $\begin{array}{c}0-0.5 \\
0.5-1 \\
1-2 \\
2-4 \\
4-8\end{array}$ & $\begin{array}{l}0.448_{-0.044}^{+0.048} \\
0.382_{-0.019}^{+0.025} \\
0.378_{-0.017}^{+0.022} \\
0.377_{-0.017}^{+0.022} \\
0.435_{-0.023}^{+0.034}\end{array}$ & $\begin{array}{c}0.15_{-0.03}^{+0.05} \\
- \\
- \\
-\end{array}$ & $\begin{array}{c}0.01_{-0.01}^{+0.41} \\
- \\
- \\
-\end{array}$ & $\begin{array}{c}0.23_{-0.03}^{+0.04} \\
- \\
- \\
-\end{array}$ & $\begin{array}{l}39.47 \\
39.65 \\
39.82 \\
39.94 \\
39.82 \\
\end{array}$ & $\begin{array}{c}39.41 \\
39.29 \\
39.38 \\
39.34 \\
0.00\end{array}$ & $\begin{array}{c}275 / 223 \\
- \\
- \\
-\end{array}$ \\
\hline NGC 3923 & $\begin{array}{c}0-0.25 \\
0.25-0.5 \\
0.5-1 \\
1-2 \\
2-4 \\
4-8 \\
\end{array}$ & $\begin{array}{l}0.613_{-0.008}^{+0.008} \\
0.559_{-0.009}^{+0.009} \\
0.479_{-0.013}^{+0.012} \\
0.491_{-0.016}^{+0.013} \\
0.562_{-0.018}^{+0.019} \\
0.471_{-0.103}^{+0.154} \\
\end{array}$ & $\begin{array}{c}0.37_{-0.04}^{+0.05} \\
- \\
- \\
- \\
-\end{array}$ & $\begin{array}{c}0.24_{-0.10}^{+0.14} \\
- \\
- \\
- \\
-\end{array}$ & $\begin{array}{c}0.37_{-0.02}^{+0.02} \\
- \\
- \\
- \\
-\end{array}$ & $\begin{array}{l}40.16 \\
40.03 \\
40.04 \\
40.00 \\
39.91 \\
39.77 \\
\end{array}$ & $\begin{array}{l}39.12 \\
39.27 \\
39.52 \\
39.65 \\
39.88 \\
39.83 \\
\end{array}$ & $\begin{array}{c}319 / 220 \\
- \\
- \\
- \\
-\end{array}$ \\
\hline NGC 4365 & $\begin{array}{l}0-1 \\
1-4 \\
\end{array}$ & $\begin{array}{l}0.522_{-0.036}^{+0.042} \\
0.624_{-0.030}^{+0.830} \\
\end{array}$ & $\begin{array}{c}1.00_{-0.32}^{+0.80} \\
- \\
\end{array}$ & $\begin{array}{c}0.96_{-0.80}^{+1.24} \\
-\end{array}$ & $\begin{array}{c}0.47_{-0.10}^{+0.11} \\
-\end{array}$ & $\begin{array}{l}39.15 \\
39.37 \\
\end{array}$ & $\begin{array}{l}39.47 \\
39.64 \\
\end{array}$ & $\begin{array}{c}177 \text { / } 190 \\
-\end{array}$ \\
\hline NGC 4382 & $\begin{array}{c}0-0.5 \\
0.5-1 \\
1-2 \\
2-4 \\
4-8\end{array}$ & $\begin{array}{l}0.404_{-0.023}^{+0.065} \\
0.384_{-0.019}^{+0.025} \\
0.349_{-0.011}^{+0.017} \\
0.315_{-0.014}^{+0.008} \\
0.266_{-0.025}^{+0.031}\end{array}$ & $\begin{array}{c}0.17_{-0.04}^{+0.07} \\
- \\
- \\
-\end{array}$ & $\begin{array}{c}0.16_{-0.16}^{+0.44} \\
- \\
- \\
-\end{array}$ & $\begin{array}{c}0.33_{-0.07}^{+0.06} \\
- \\
- \\
-\end{array}$ & $\begin{array}{l}39.36 \\
39.53 \\
39.76 \\
39.80 \\
39.70\end{array}$ & $\begin{array}{l}39.20 \\
39.09 \\
39.07 \\
39.45 \\
39.43\end{array}$ & $\begin{array}{c}244 \text { / } 211 \\
- \\
- \\
-\end{array}$ \\
\hline
\end{tabular}


R. Nagino and K. Matsushita: Gravitational potential and X-ray luminosities of early-type galaxies, Online Material p 2

Table 3. continued.

\begin{tabular}{|c|c|c|c|c|c|c|c|c|}
\hline Galaxy & $\begin{array}{l}\text { Ring } \\
\left(r_{\mathrm{e}}\right)\end{array}$ & $\begin{array}{c}k T \\
(\mathrm{keV})\end{array}$ & $\begin{array}{c}\mathrm{O} \\
\text { (solar) } \\
\end{array}$ & $\begin{array}{c}\mathrm{Si} \\
\text { (solar) }\end{array}$ & $\begin{array}{c}\mathrm{Fe} \\
\text { (solar) }\end{array}$ & $\begin{array}{c}\log L_{\text {ISM }} \\
(\mathrm{erg} / \mathrm{s})\end{array}$ & $\begin{array}{c}\log L_{\text {hard }} \\
(\mathrm{erg} / \mathrm{s})\end{array}$ & $\chi^{2} /$ d.o.f. \\
\hline \multirow[t]{8}{*}{ NGC 4472} & $0-0.25$ & 0.782 & 0.77 & 1.15 & 0.92 & 40.61 & 39.53 & $782 / 272$ \\
\hline & $0.25-0.5$ & 0.903 & 0.75 & 0.97 & 0.77 & 40.47 & 39.49 & 964 / 216 \\
\hline & $0.5-0.75$ & 0.995 & 1.01 & 1.53 & 1.11 & 40.40 & 39.58 & $667 / 229$ \\
\hline & $0.75-1$ & 1.031 & 0.86 & 1.68 & 1.10 & 40.36 & 39.52 & $541 / 195$ \\
\hline & $1-1.5$ & 1.044 & 0.94 & 1.76 & 1.22 & 40.59 & 39.76 & 808 / 194 \\
\hline & $1.5-2$ & 1.160 & 0.58 & 1.38 & 1.06 & 40.50 & 39.65 & $625 / 186$ \\
\hline & $2-3$ & 1.227 & 0.58 & 1.35 & 1.00 & 40.61 & 39.78 & 427 / 193 \\
\hline & $3-4$ & $1.270_{-0.013}^{+0.012}$ & $0.39_{-0.14}^{+0.15}$ & $1.27_{-0.16}^{+0.20}$ & $0.67_{-0.05}^{+0.05}$ & 40.38 & 39.42 & $201 / 115$ \\
\hline \multirow[t]{4}{*}{ NGC 4477} & $0-1$ & $0.446_{-0.027}^{+0.023}$ & $0.25_{-0.05}^{+0.04}$ & $1.00^{*}$ & $0.17_{-0.02}^{+0.02}$ & 39.71 & 39.01 & 97 / 79 \\
\hline & $1-2$ & $0.452_{-0.029}^{+0.027}$ & -0.05 & - & $-^{-0.02}$ & 39.56 & 38.60 & - \\
\hline & $2-4$ & $0.273_{-0.193}^{+0.279}$ & - & - & - & 38.67 & 39.32 & - \\
\hline & $4-8$ & $0.122_{-0.041}^{+0.241}$ & - & - & - & 38.99 & 39.28 & - \\
\hline \multirow[t]{3}{*}{ NGC 4526} & $0-0.5$ & $0.353_{-0.024}^{+0.029}$ & $0.29_{-0.06}^{+0.08}$ & $1.00^{*}$ & $0.40_{-0.06}^{+0.06}$ & 39.21 & 39.29 & 77 / 117 \\
\hline & $0.5-1$ & $0.322_{-0.024}^{+0.035}$ & $-^{-0.00}$ & - & $\underline{-}^{-0.06}$ & 38.92 & 38.92 & - \\
\hline & $1-4$ & $0.280_{-0.031}^{+0.034}$ & - & - & - & 38.97 & 39.20 & - \\
\hline \multirow[t]{4}{*}{ NGC 4552} & $0-0.5$ & $0.679_{-0.011}^{+0.020}$ & $0.52_{-0.06}^{+0.05}$ & $0.56_{-0.14}^{+0.13}$ & $0.41_{-0.02}^{+0.02}$ & 40.21 & 39.81 & $275 / 182$ \\
\hline & $0.5-1$ & $0.582_{-0.009}^{+0.010}$ & $\underline{-}^{-0.00}$ & $-^{-0.14}$ & $-^{-0.02}$ & 40.08 & 39.54 & - \\
\hline & $1-2$ & $0.500_{-0.014}^{+0.009}$ & - & - & - & 39.97 & 39.63 & - \\
\hline & $2-4$ & $0.528_{-0.034}^{+0.0145}$ & - & - & - & 39.66 & 39.63 & - \\
\hline \multirow[t]{9}{*}{ NGC 4636} & $0-0.25$ & 0.561 & 0.45 & 0.59 & 0.46 & 40.63 & 38.95 & $881 / 335$ \\
\hline & $0.25-0.5$ & $0.599_{-0.002}^{+0.002}$ & $0.61_{-0.02}^{+0.03}$ & $0.88_{-0.05}^{+0.06}$ & $0.68_{-0.02}^{+0.02}$ & 40.66 & 38.94 & $656 / 350$ \\
\hline & $0.5-0.75$ & $0.653_{-0.002}^{+0.002}$ & $0.93_{-0.05}^{+0.02}$ & $\begin{array}{r}-0.05 \\
1.36_{-0.09}^{+0.08}\end{array}$ & $0.96_{-0.03}^{+0.02}$ & 40.65 & 39.15 & 549 / 293 \\
\hline & $0.75-1$ & $0.706_{-0.002}^{+0.002}$ & $0.91_{-0.06}^{+0.05}$ & $1.51_{-0.06}^{+0.09}$ & $0.99_{-0.04}^{+0.03}$ & 40.52 & 39.17 & 417 / 252 \\
\hline & $1-1.5$ & $0.742_{-0.002}^{-0.002}$ & $0.82_{-0.06}^{+0.06}$ & $1.45_{-0.09}^{+0.06}$ & $1.05_{-0.03}^{-0.04}$ & 40.61 & 39.47 & 535 / 297 \\
\hline & $1.5-2$ & $\begin{array}{r}-0.002 \\
0.777_{-0.003}^{+0.003}\end{array}$ & $0.79_{-0.09}^{+0.06}$ & $\begin{array}{r}-0.09 \\
1.37_{-0.07}^{+0.06}\end{array}$ & $\begin{array}{r}-0.03 \\
1.07_{-0.04}^{+0.05}\end{array}$ & 40.46 & 39.47 & $306 / 254$ \\
\hline & $2-3$ & $0.806_{-0.003}^{-0.003}$ & $0.87_{-0.11}^{-0.09}$ & $1.32_{-0.12}^{+0.07}$ & $1.12_{-0.05}^{-0.04}$ & 40.58 & 39.65 & $390 / 288$ \\
\hline & $3-4$ & $0.818_{-0.005}^{+0.005}$ & $0.98_{-0.22}^{+0.25}$ & $1.01_{-0.22}^{+0.121}$ & $0.99_{-0.08}^{+0.05}$ & 40.27 & 39.53 & $263 / 227$ \\
\hline & $4-6$ & $0.845_{-0.011}^{+0.011}$ & $0.83_{-0.22}^{+0.36}$ & $0.28_{-0.28}^{+0.32}$ & $0.43_{-0.07}^{+0.08}$ & 40.21 & 37.80 & $119 / 111$ \\
\hline \multirow[t]{8}{*}{ NGC 4649} & $0-0.25$ & 0.810 & 1.11 & 1.81 & 1.50 & 40.42 & 39.47 & $757 / 343$ \\
\hline & $0.25-0.5$ & $0.782_{-0.002}^{+0.002}$ & $1.24_{-0.11}^{+0.13}$ & $2.29_{-0.18}^{+0.20}$ & $1.55_{-0.08}^{+0.09}$ & 40.34 & 39.43 & $410 / 222$ \\
\hline & $0.5-0.75$ & $0.791_{-0.003}^{+0.003}$ & $1.23_{-0.17}^{+0.20}$ & $2.18_{-0.24}^{+0.29}$ & $1.60_{-0.09}^{+0.10}$ & 40.10 & 39.33 & $259 / 158$ \\
\hline & $0.75-1$ & $0.805_{-0.004}^{+0.003}$ & $0.82_{-0.13}^{-0.17}$ & $1.65_{-0.21}^{-0.24}$ & $1.15_{-0.06}^{-0.09}$ & 39.95 & 39.29 & 187 / 119 \\
\hline & $1-1.5$ & $0.821_{-0.004}^{+0.004}$ & $1.13_{-0.17}^{-0.13}$ & $2.12_{-0.25}^{-0.21}$ & $1.34_{-0.05}^{-0.06}$ & 40.10 & 39.70 & $258 / 188$ \\
\hline & $1.5-2$ & $0.828_{-0.005}^{+0.004}$ & $1.09_{-0.22}^{+0.32}$ & $2.34_{-0.37}^{+0.52}$ & $1.28_{-0.04}^{+0.05}$ & 39.88 & 39.53 & $163 / 131$ \\
\hline & $2-4$ & $0.836_{-0.008}^{+0.000}$ & $0.36_{-0.10}^{+0.12}$ & $0.40_{-0.15}^{+0.16}$ & $0.34_{-0.04}^{+0.04}$ & 40.05 & 39.88 & $250 / 193$ \\
\hline & $4-6$ & $0.863_{-0.054}^{+0.0119}$ & $0.50_{-0.36}^{+1.02}$ & $0.00_{-0.00}^{+0.25}$ & $0.13_{-0.05}^{+0.04}$ & 39.37 & 39.46 & $72 / 53$ \\
\hline \multirow[t]{8}{*}{ NGC 5044} & $0-0.5$ & $0.771_{-0.004}^{+0.004}$ & $0.61_{-0.06}^{+0.07}$ & $0.82_{-0.08}^{+0.09}$ & $0.65_{-0.03}^{+0.03}$ & 41.48 & 40.14 & $214 / 126$ \\
\hline & $0.5-1$ & $0.798_{-0.003}^{+0.002}$ & $0.69_{-0.05}^{+0.06}$ & $0.99_{-0.07}^{+0.07}$ & $0.78_{-0.03}^{+0.03}$ & 41.76 & 40.27 & $609 / 356$ \\
\hline & $1-1.5$ & $0.833_{-0.003}^{+0.002}$ & $0.74_{-0.06}^{+0.06}$ & $1.05_{-0.07}^{+0.08}$ & $0.75_{-0.03}^{+0.03}$ & 41.74 & 40.24 & $978 / 520$ \\
\hline & $1.5-2$ & $0.929_{-0.005}^{+0.008}$ & $0.54_{-0.05}^{+0.05}$ & $0.80_{-0.06}^{+0.06}$ & $0.67_{-0.02}^{+0.02}$ & 41.68 & 40.22 & $676 / 349$ \\
\hline & $2-3$ & $1.004_{-0.003}^{+0.003}$ & $0.50_{-0.04}^{+0.05}$ & $0.80_{-0.05}^{+0.09}$ & $0.68_{-0.02}^{+0.02}$ & 41.86 & 40.43 & 787 / 491 \\
\hline & $3-4$ & $1.196_{-0.007}^{+0.007}$ & $0.41_{-0.06}^{+0.04}$ & $0.64_{-0.05}^{+0.05}$ & $0.65_{-0.03}^{+0.02}$ & 41.65 & 40.00 & 334 / 194 \\
\hline & $4-6$ & $1.247_{-0.006}^{+0.006}$ & $0.33_{-0.06}^{+0.06}$ & $0.68_{-0.05}^{+0.05}$ & $0.62_{-0.02}^{+0.03}$ & 41.79 & 40.50 & $316 / 249$ \\
\hline & $6-8$ & $1.231_{-0.013}^{+0.012}$ & $0.54_{-0.11}^{+0.09}$ & $0.57_{-0.09}^{+0.03}$ & $0.47_{-0.03}^{+0.02}$ & 41.53 & 40.48 & $192 / 176$ \\
\hline \multirow[t]{4}{*}{ NGC 5322} & $0-1$ & $0.440_{-0.056}^{+0.038}$ & $0.13_{-0.04}^{+0.06}$ & $1.00^{*}$ & $0.15_{-0.03}^{+0.02}$ & 39.93 & 39.33 & 67 / 71 \\
\hline & $1-2$ & $0.373_{-0.054}^{+0.059}$ & $-^{-0.04}$ & - & $-^{-0.03}$ & 39.37 & 39.29 & - \\
\hline & $2-4$ & $0.391_{-0.088}^{+0.034}$ & - & - & - & 39.46 & 39.39 & - \\
\hline & $4-8$ & $0.306_{-0.060}^{+0.068}$ & - & - & - & 39.73 & 39.60 & - \\
\hline \multirow[t]{7}{*}{ NGC 5846} & $0-0.5$ & $0.652_{-0.005}^{+0.005}$ & $0.72_{-0.05}^{+0.06}$ & $0.77_{-0.08}^{+0.09}$ & $0.68_{-0.02}^{+0.02}$ & 41.05 & 39.87 & $803 / 450$ \\
\hline & $0.5-1$ & $0.644_{-0.006}^{+0.005}$ & $-^{-0.05}$ & $-^{-0.08}$ & -0.02 & 41.06 & 39.76 & - \\
\hline & $1-1.5$ & $0.699_{-0.006}^{+0.006}$ & - & - & - & 40.97 & 39.75 & - \\
\hline & $1.5-2$ & $0.749_{-0.007}^{+0.006}$ & - & - & - & 40.91 & 39.79 & - \\
\hline & $2-3$ & $0.838_{-0.007}^{+0.007}$ & - & - & - & 40.91 & 39.94 & - \\
\hline & $3-4$ & $1.160_{-0.035}^{+0.0032}$ & - & - & - & 40.56 & 39.38 & - \\
\hline & $4-8$ & $1.189_{-0.070}^{+0.035}$ & - & - & - & 40.70 & 40.30 & - \\
\hline
\end{tabular}

${ }^{a}$ The inner and outer radii to integrated the spectrum.

${ }^{b} k T$ is ISM temperature. $L_{\mathrm{ISM}}$ and $L_{\mathrm{hard}}$ are the $\mathrm{X}$-ray luminosities of the thermal emission and the non thermal emission in the range of $0.3-2.0 \mathrm{keV}$, respectively.

${ }^{*}$ Fixed to the value. 
R. Nagino and K. Matsushita: Gravitational potential and X-ray luminosities of early-type galaxies, Online Material p 3

Table 4. Spectral fitting results of the deprojected annular spectra.

\begin{tabular}{|c|c|c|c|c|c|c|c|c|}
\hline Galaxy & $\begin{array}{c}\operatorname{Ring}^{a} \\
\left(r_{\mathrm{e}}\right)\end{array}$ & $\begin{array}{c}k T^{b} \\
(\mathrm{keV})\end{array}$ & $\begin{array}{c}\mathrm{O} \\
\text { (solar) }\end{array}$ & $\begin{array}{c}\mathrm{Si} \\
\text { (solar) }\end{array}$ & $\begin{array}{c}\mathrm{Fe} \\
\text { (solar) }\end{array}$ & $\begin{array}{c}\log L_{\mathrm{ISM}}{ }^{b} \\
(\mathrm{erg} / \mathrm{s})\end{array}$ & $\begin{array}{c}\log L_{\mathrm{hard}}{ }^{b} \\
(\mathrm{erg} / \mathrm{s})\end{array}$ & $\chi^{2} /$ d.o.f. \\
\hline IC 1459 & $\begin{array}{c}0-0.5 \\
0.5-1 \\
1-2 \\
2-4 \\
4-8\end{array}$ & $\begin{array}{l}0.616_{-0.027}^{+0.028} \\
0.642_{-0.065}^{+0.058} \\
0.557_{-0.059}^{+0.047} \\
0.523_{-0.068}^{+0.085} \\
0.644_{-0.032}^{+0.031} \\
\end{array}$ & $\begin{array}{c}0.35_{-0.13}^{+0.27} \\
- \\
- \\
-\end{array}$ & $\begin{array}{c}0.09_{-0.09}^{+0.51} \\
- \\
- \\
-\end{array}$ & $\begin{array}{c}0.28_{-0.03}^{+0.04} \\
- \\
- \\
-\end{array}$ & $\begin{array}{l}39.54 \\
39.20 \\
39.41 \\
39.38 \\
39.72 \\
\end{array}$ & $\begin{array}{l}40.02 \\
39.55 \\
39.52 \\
39.18 \\
39.28 \\
\end{array}$ & $\begin{array}{c}174 \text { / } 197 \\
- \\
- \\
-\end{array}$ \\
\hline NGC 720 & $\begin{array}{l}0-1 \\
1-2 \\
2-4 \\
4-8 \\
\end{array}$ & $\begin{array}{l}0.606_{-0.015}^{+0.015} \\
0.570_{-0.034}^{+0.027} \\
0.566_{-0.022}^{+0.022} \\
0.454_{-0.032}^{+0.031}\end{array}$ & $\begin{array}{c}0.67_{-0.19}^{+0.27} \\
- \\
- \\
-\end{array}$ & $\begin{array}{c}1.00^{*} \\
- \\
- \\
-\end{array}$ & $\begin{array}{c}0.70_{-0.06}^{+0.07} \\
- \\
-\end{array}$ & $\begin{array}{l}39.88 \\
39.84 \\
39.97 \\
40.00 \\
\end{array}$ & $\begin{array}{l}39.37 \\
39.32 \\
39.62 \\
39.67 \\
\end{array}$ & $\begin{array}{c}108 / 97 \\
- \\
-\end{array}$ \\
\hline NGC 1316 & $\begin{array}{c}0-0.25 \\
0.25-0.5 \\
0.5-1 \\
1-2 \\
2-4 \\
4-8\end{array}$ & $\begin{array}{l}0.748_{-0.007}^{+0.007} \\
0.657_{-0.019}^{+0.019} \\
0.586_{-0.013}^{+0.014} \\
0.702_{-0.041}^{+0.025} \\
0.639_{-0.048}^{+0.115} \\
0.740_{-0.031}^{+0.028}\end{array}$ & $\begin{array}{c}0.54_{-0.06}^{+0.08} \\
- \\
- \\
- \\
-\end{array}$ & $\begin{array}{c}0.22_{-0.09}^{+0.11} \\
- \\
- \\
- \\
-\end{array}$ & $\begin{array}{c}0.44_{-0.01}^{+0.02} \\
- \\
- \\
- \\
-\end{array}$ & $\begin{array}{l}39.99 \\
39.68 \\
39.96 \\
39.83 \\
39.68 \\
40.17\end{array}$ & $\begin{array}{l}39.39 \\
39.30 \\
39.73 \\
39.52 \\
39.63 \\
40.10\end{array}$ & $\begin{array}{c}586 / 615 \\
- \\
- \\
- \\
-\end{array}$ \\
\hline NGC 1332 & $\begin{array}{c}0-0.5 \\
0.5-1 \\
1-2 \\
2-4 \\
4-8 \\
\end{array}$ & $\begin{array}{l}0.615_{-0.008}^{+0.008} \\
0.598_{-0.021}^{+0.021} \\
0.468_{-0.027}^{+0.029} \\
0.465_{-0.024}^{+0.025} \\
0.511_{-0.032}^{+0.033} \\
\end{array}$ & $\begin{array}{c}0.74_{-0.12}^{+0.16} \\
- \\
- \\
-\end{array}$ & $\begin{array}{c}0.82_{-0.27}^{+0.29} \\
- \\
- \\
-\end{array}$ & $\begin{array}{c}0.65_{-0.02}^{+0.05} \\
- \\
- \\
- \\
-\end{array}$ & $\begin{array}{l}39.62 \\
39.31 \\
39.30 \\
39.42 \\
39.26 \\
\end{array}$ & $\begin{array}{l}39.06 \\
39.06 \\
39.12 \\
39.27 \\
39.15 \\
\end{array}$ & $\begin{array}{c}266 / 260 \\
- \\
- \\
-\end{array}$ \\
\hline NGC 1395 & $\begin{array}{c}0-0.5 \\
0.5-1 \\
1-2 \\
2-4 \\
4-8 \\
\end{array}$ & $\begin{array}{l}0.592_{-0.019}^{+0.019} \\
0.520_{-0.035}^{+0.050} \\
0.698_{-0.028}^{+0.029} \\
0.743_{-0.019}^{+0.019} \\
0.771_{-0.017}^{+0.018} \\
\end{array}$ & $\begin{array}{c}0.80_{-0.12}^{+0.51} \\
- \\
- \\
- \\
-\end{array}$ & $\begin{array}{c}1.00^{*} \\
- \\
- \\
- \\
-\end{array}$ & $\begin{array}{c}0.87_{-0.05}^{+0.06} \\
- \\
- \\
- \\
-\end{array}$ & $\begin{array}{l}39.41 \\
39.54 \\
39.78 \\
39.99 \\
40.13 \\
\end{array}$ & $\begin{array}{l}39.19 \\
39.23 \\
39.71 \\
39.19 \\
40.02 \\
\end{array}$ & $\begin{array}{c}132 / 173 \\
- \\
- \\
-\end{array}$ \\
\hline NGC 1399 & $\begin{array}{c}0-0.5 \\
0.5-1 \\
1-2 \\
2-4 \\
4-6 \\
6-8 \\
\end{array}$ & $\begin{array}{l}0.826_{-0.011}^{+0.010} \\
0.934_{-0.028}^{+0.034} \\
0.995_{-0.034}^{+0.039} \\
1.332_{-0.026}^{+0.033} \\
1.352_{-0.030}^{+0.062} \\
1.461_{-0.109}^{+0.08} \\
\end{array}$ & $\begin{array}{c}0.58_{-0.25}^{+0.36} \\
- \\
- \\
- \\
- \\
\end{array}$ & $\begin{array}{c}0.89_{-0.38}^{+0.37} \\
- \\
- \\
- \\
-\end{array}$ & $\begin{array}{c}1.18_{-0.09}^{+0.10} \\
- \\
- \\
- \\
-\end{array}$ & $\begin{array}{l}40.36 \\
40.25 \\
40.15 \\
40.62 \\
40.59 \\
40.68 \\
\end{array}$ & $\begin{array}{l}39.47 \\
39.62 \\
39.86 \\
39.84 \\
39.85 \\
40.35 \\
\end{array}$ & $\begin{array}{c}158 / 163 \\
- \\
- \\
- \\
-\end{array}$ \\
\hline NGC 3923 & $\begin{array}{c}0-0.25 \\
0.25-0.5 \\
0.5-1 \\
1-2 \\
2-4 \\
4-8 \\
\end{array}$ & $\begin{array}{l}0.627_{-0.011}^{+0.012} \\
0.575_{-0.017}^{+0.0117} \\
0.482_{-0.025}^{+0.028} \\
0.514_{-0.029}^{+0.042} \\
0.490_{-0.037}^{+0.033} \\
0.367_{-0.023}^{+0.028} \\
\end{array}$ & $\begin{array}{c}0.46_{-0.10}^{+0.11} \\
- \\
- \\
- \\
-\end{array}$ & $\begin{array}{l}1.00^{*} \\
- \\
- \\
- \\
- \\
\end{array}$ & $\begin{array}{c}0.47_{-0.02}^{+0.02} \\
- \\
- \\
- \\
-\end{array}$ & $\begin{array}{l}40.04 \\
39.99 \\
40.03 \\
39.97 \\
39.87 \\
40.06 \\
\end{array}$ & $\begin{array}{l}38.80 \\
39.14 \\
39.42 \\
39.35 \\
39.95 \\
39.82 \\
\end{array}$ & $\begin{array}{c}194 / 212 \\
- \\
- \\
- \\
-\end{array}$ \\
\hline $\begin{array}{l}\text { NGC } 4472 \\
(2 T \text { model })^{c}\end{array}$ & $0-0.25$ & $\begin{array}{l}0.738_{-0.013}^{+0.013} \\
1.263_{-0.104}^{+0.090}\end{array}$ & $\begin{array}{c}0.70_{-0.12}^{+0.12} \\
-\end{array}$ & $\begin{array}{c}1.19_{-0.16}^{+0.12} \\
-\end{array}$ & $\begin{array}{c}1.13_{-0.08}^{+0.09} \\
-\end{array}$ & $\begin{array}{c}40.49 \\
-\end{array}$ & $\begin{array}{c}39.39 \\
-\end{array}$ & $\begin{array}{c}237 \text { / } 159 \\
-\end{array}$ \\
\hline NGC 4472 & $\begin{array}{c}0-0.25 \\
0.25-0.5 \\
0.5-0.75 \\
0.75-1 \\
1-1.5 \\
1.5-2 \\
2-3 \\
3-4 \\
4-6 \\
6-8 \\
\end{array}$ & $\begin{array}{l}0.764_{-0.003}^{+0.003} \\
0.784_{-0.007}^{+0.007} \\
0.920_{-0.019}^{+0.025} \\
1.013_{-0.021}^{+0.019} \\
1.020_{-0.010}^{+0.011} \\
1.045_{-0.013}^{+0.012} \\
1.210_{-0.017}^{+0.016} \\
1.232_{-0.044}^{+0.037} \\
1.334_{-0.015}^{+0.014} \\
1.392_{-0.044}^{+0.048} \\
\end{array}$ & $\begin{array}{l}0.72_{-0.09}^{+0.08} \\
0.76_{-0.18}^{+0.24} \\
1.42_{-0.88}^{+0.65} \\
0.60_{-0.34}^{+0.62} \\
0.88_{-0.26}^{+0.47} \\
0.34_{-0.31}^{+0.38} \\
0.38_{-0.26}^{+0.36} \\
0.14_{-0.14}^{+0.43} \\
0.05_{-0.05}^{+0.16} \\
0.00_{-0.00}^{+0.09} \\
\end{array}$ & $\begin{array}{l}1.15_{-0.13}^{+0.12} \\
1.04_{-0.26}^{+0.29} \\
1.51_{-0.63}^{+0.36} \\
1.27_{-0.49}^{+0.76} \\
1.51_{-0.38}^{+0.51} \\
1.17_{-0.40}^{+0.54} \\
1.05_{-0.39}^{+0.31} \\
0.89_{-0.36}^{+0.43} \\
0.99_{-0.16}^{+0.18} \\
0.39_{-0.10}^{+0.10} \\
\end{array}$ & $\begin{array}{l}1.01_{-0.05}^{+0.05} \\
0.87_{-0.09}^{+0.11} \\
1.35_{-0.28}^{+0.26} \\
0.96_{-0.15}^{+0.21} \\
1.21_{-0.14}^{+0.18} \\
1.04_{-0.13}^{+0.26} \\
1.08_{-0.13}^{+0.11} \\
0.51_{-0.13}^{+0.11} \\
0.47_{-0.05}^{+0.07} \\
0.34_{-0.04}^{+0.03} \\
\end{array}$ & $\begin{array}{l}40.48 \\
40.33 \\
40.24 \\
40.26 \\
40.52 \\
40.49 \\
40.65 \\
40.50 \\
40.79 \\
40.68 \\
\end{array}$ & $\begin{array}{l}39.47 \\
39.41 \\
39.62 \\
39.65 \\
39.77 \\
39.86 \\
39.99 \\
39.36 \\
39.97 \\
40.04 \\
\end{array}$ & $\begin{array}{c}281 \text { / } 161 \\
95 / 88 \\
44 \text { / } 61 \\
28 \text { / } 66 \\
53 \text { / } 68 \\
49 \text { / } 67 \\
52 \text { / } 91 \\
22 / 48 \\
98 \text { / } 107 \\
118 \text { / } 102 \\
\end{array}$ \\
\hline NGC 4552 & $\begin{array}{c}0-0.5 \\
0.5-1 \\
1-2 \\
2-4\end{array}$ & $\begin{array}{l}0.708_{-0.013}^{+0.013} \\
0.610_{-0.016}^{+0.016} \\
0.500_{-0.023}^{+0.021} \\
0.469_{-0.037}^{+0.039}\end{array}$ & $\begin{array}{c}0.53_{-0.10}^{+0.07} \\
- \\
-\end{array}$ & $\begin{array}{c}0.89_{-0.24}^{+0.27} \\
- \\
-\end{array}$ & $\begin{array}{c}0.46_{-0.03}^{+0.05} \\
- \\
-\end{array}$ & $\begin{array}{l}40.08 \\
40.07 \\
40.01 \\
39.73\end{array}$ & $\begin{array}{l}39.72 \\
39.43 \\
39.65 \\
39.73\end{array}$ & $\begin{array}{c}162 \text { / } 159 \\
- \\
-\end{array}$ \\
\hline
\end{tabular}


R. Nagino and K. Matsushita: Gravitational potential and X-ray luminosities of early-type galaxies, Online Material p 4

Table 4. continued.

\begin{tabular}{|c|c|c|c|c|c|c|c|c|}
\hline Galaxy & $\begin{array}{l}\text { Ring } \\
\left(r_{\mathrm{e}}\right)\end{array}$ & $\begin{array}{c}k T \\
(\mathrm{keV})\end{array}$ & $\begin{array}{c}\mathrm{O} \\
\text { (solar) }\end{array}$ & $\begin{array}{c}\mathrm{Si} \\
\text { (solar) }\end{array}$ & $\begin{array}{c}\mathrm{Fe} \\
\text { (solar) }\end{array}$ & $\begin{array}{c}\log L_{\mathrm{ISM}} \\
(\mathrm{erg} / \mathrm{s})\end{array}$ & $\begin{array}{c}\log L_{\text {hard }} \\
(\mathrm{erg} / \mathrm{s})\end{array}$ & $\chi^{2} /$ d.o.f. \\
\hline $\begin{array}{l}\text { NGC } 4636 \\
\text { (2T model) }\end{array}$ & $0-0.25$ & $\begin{array}{l}0.438_{-0.014}^{+0.045} \\
0.657_{-0.042}^{+0.042}\end{array}$ & $\begin{array}{c}0.40_{-0.03}^{+0.03} \\
-\end{array}$ & $0.68_{-0.09}^{+0.10}$ & $\begin{array}{c}0.49_{-0.03}^{+0.04} \\
-\end{array}$ & $\begin{array}{c}40.45 \\
-\end{array}$ & $\begin{array}{c}38.89 \\
-\end{array}$ & $\begin{array}{c}416 / 338 \\
-\end{array}$ \\
\hline NGC 4636 & $\begin{array}{c}0-0.25 \\
0.25-0.5 \\
0.5-0.75 \\
0.75-1 \\
1-1.5 \\
1.5-2 \\
2-3 \\
3-4 \\
4-6 \\
6-8\end{array}$ & $\begin{array}{l}0.526_{-0.005}^{+0.005} \\
0.520_{-0.008}^{+0.009} \\
0.618_{-0.006}^{+0.006} \\
0.671_{-0.007}^{+0.007} \\
0.711_{-0.007}^{+0.007} \\
0.749_{-0.012}^{+0.013} \\
0.799_{-0.007}^{+0.007} \\
0.808_{-0.015}^{+0.015} \\
0.839_{-0.019}^{+0.018} \\
0.783_{-0.022}^{+0.022}\end{array}$ & $\begin{array}{c}0.42_{-0.03}^{+0.03} \\
0.47_{-0.06}^{+0.06} \\
0.76_{-0.08}^{+0.25} \\
1.02_{-0.26}^{+0.35} \\
0.77_{-0.17}^{+0.37} \\
0.94_{-0.54}^{+0.97} \\
0.56_{-0.16}^{+0.85} \\
1.10_{-0.76}^{+27.28} \\
0.64_{-0.29}^{+0.55} \\
0.19_{-0.08}^{+0.08}\end{array}$ & $\begin{array}{l}0.57_{-0.08}^{+0.09} \\
0.91_{-0.24}^{+0.25} \\
0.97_{-0.23}^{+0.43} \\
1.60_{-0.39}^{+0.67} \\
1.33_{-0.35}^{+0.41} \\
1.28_{-0.78}^{+1.01} \\
0.95_{-0.34}^{+0.65} \\
0.77_{-0.77}^{+0.93} \\
0.00_{-0.00}^{+0.58} \\
0.04_{-0.04}^{+0.16}\end{array}$ & $\begin{array}{l}0.41_{-0.02}^{+0.02} \\
0.56_{-0.05}^{+0.06} \\
0.89_{-0.06}^{+0.16} \\
1.11_{-0.15}^{+0.20} \\
1.04_{-0.10}^{+0.17} \\
1.13_{-0.26}^{+0.23} \\
0.84_{-0.08}^{+0.21} \\
1.78_{-0.67}^{+1.58} \\
0.39_{-0.08}^{+0.23} \\
0.12_{-0.02}^{+0.01}\end{array}$ & \begin{tabular}{l|}
40.45 \\
40.48 \\
40.61 \\
40.54 \\
40.58 \\
40.46 \\
40.68 \\
40.38 \\
40.47 \\
40.68
\end{tabular} & $\begin{array}{c}38.81 \\
0.00 \\
39.21 \\
39.34 \\
39.54 \\
39.57 \\
39.59 \\
39.91 \\
0.00 \\
39.42\end{array}$ & $\begin{array}{c}448 \text { / } 340 \\
189 \text { / } 222 \\
125 / 192 \\
95 / 180 \\
74 / 156 \\
33 / 122 \\
38 / 106 \\
22 / 77 \\
21 / 48 \\
101 / 83\end{array}$ \\
\hline $\begin{array}{l}\text { NGC } 4649 \\
\text { (2T model) } \\
\end{array}$ & $0-0.25$ & $\begin{array}{l}0.810_{-0.004}^{+0.003} \\
2.766_{-0.199}^{+0.198} \\
\end{array}$ & $\begin{array}{c}0.96_{-0.09}^{+0.19} \\
-\end{array}$ & $\begin{array}{c}1.38_{-0.16}^{+0.26} \\
-\end{array}$ & $\begin{array}{c}1.73_{-0.10}^{+0.21} \\
-\end{array}$ & $\begin{array}{c}40.32 \\
-\end{array}$ & $\begin{array}{c}0.00 \\
-\end{array}$ & $\begin{array}{c}265 \text { / } 197 \\
-\end{array}$ \\
\hline NGC 4649 & $\begin{array}{c}0-0.25 \\
0.25-0.5 \\
0.5-0.75 \\
0.75-1 \\
1-1.5 \\
1.5-2 \\
2-4 \\
4-6 \\
\end{array}$ & $\begin{array}{l}0.819_{-0.003}^{+0.003} \\
0.776_{-0.005}^{+0.004} \\
0.778_{-0.009}^{+0.009} \\
0.792_{-0.015}^{+0.014} \\
0.816_{-0.009}^{+0.009} \\
0.830_{-0.013}^{+0.012} \\
0.913_{-0.023}^{+0.021} \\
0.864_{-0.041}^{+0.063}\end{array}$ & $\begin{array}{l}1.11_{-0.14}^{+0.17} \\
1.21_{-0.21}^{+0.28} \\
1.75_{-0.61}^{+1.69} \\
0.55_{-0.32}^{+0.58} \\
1.04_{-0.28}^{+0.90} \\
0.49_{-0.32}^{+0.82} \\
0.18_{-0.12}^{+0.13} \\
0.00_{-0.00}^{+0.11} \\
\end{array}$ & $\begin{array}{l}1.63_{-0.18}^{+0.21} \\
2.29_{-0.31}^{+0.43} \\
3.09_{-0.90}^{+0.96} \\
1.04_{-0.68}^{+0.74} \\
1.77_{-0.53}^{+1.01} \\
1.00_{-1.00}^{+1.00} \\
0.42_{-0.17}^{+0.16} \\
0.00_{-0.00}^{+0.14}\end{array}$ & $\begin{array}{l}1.54_{-0.05}^{+0.12} \\
1.61_{-0.16}^{+0.22} \\
2.29_{-0.49}^{+0.53} \\
0.98_{-0.15}^{+0.24} \\
1.22_{-0.11}^{+0.24} \\
1.02_{-0.09}^{+0.13} \\
0.26_{-0.03}^{+0.04} \\
0.07_{-0.02}^{+0.04}\end{array}$ & $\begin{array}{l}40.27 \\
40.31 \\
40.08 \\
39.92 \\
40.14 \\
39.97 \\
40.20 \\
39.81 \\
\end{array}$ & $\begin{array}{l}39.38 \\
39.40 \\
39.23 \\
39.25 \\
39.68 \\
39.70 \\
39.82 \\
39.79 \\
\end{array}$ & $\begin{array}{c}375 \text { / } 199 \\
189 \text { / } 206 \\
83 \text { / } 147 \\
68 \text { / } 108 \\
96 / 186 \\
77 \text { / } 166 \\
167 \text { / } 206 \\
149 \text { / } 157 \\
\end{array}$ \\
\hline NGC 5044 & $\begin{array}{c}0-0.5 \\
0.5-1 \\
1-1.5 \\
1.5-2 \\
2-3 \\
3-4 \\
4-6 \\
6-8 \\
\end{array}$ & $\begin{array}{l}0.729_{-0.012}^{+0.013} \\
0.762_{-0.007}^{+0.007} \\
0.794_{-0.008}^{+0.008} \\
0.833_{-0.009}^{+0.009} \\
0.944_{-0.004}^{+0.008} \\
1.138_{-0.024}^{+0.026} \\
1.248_{-0.010}^{+0.011} \\
1.224_{-0.015}^{+0.014} \\
\end{array}$ & $\begin{array}{l}0.57_{-0.17}^{+0.16} \\
0.62_{-0.15}^{+0.18} \\
0.81_{-0.20}^{+0.34} \\
0.67_{-0.23}^{+0.34} \\
0.54_{-0.12}^{+0.13} \\
0.31_{-0.14}^{+0.18} \\
0.23_{-0.10}^{+0.11} \\
0.63_{-0.13}^{+0.14} \\
\end{array}$ & $\begin{array}{l}0.44_{-0.28}^{+0.29} \\
0.76_{-0.21}^{+0.25} \\
1.29_{-0.29}^{+0.46} \\
0.95_{-0.29}^{+0.22} \\
0.82_{-0.12}^{+0.14} \\
0.42_{-0.14}^{+0.15} \\
0.72_{-0.09}^{+0.09} \\
0.56_{-0.09}^{+0.10} \\
\end{array}$ & $\begin{array}{l}0.53_{-0.07}^{+0.07} \\
0.83_{-0.08}^{+0.10} \\
0.92_{-0.16}^{+0.16} \\
0.84_{-0.11}^{+0.11} \\
0.76_{-0.06}^{+0.04} \\
0.58_{-0.07}^{+0.06} \\
0.70_{-0.05}^{+0.05} \\
0.48_{-0.04}^{+0.04}\end{array}$ & $\begin{array}{l}41.09 \\
41.57 \\
41.65 \\
41.62 \\
41.88 \\
41.67 \\
41.84 \\
41.78 \\
\end{array}$ & $\begin{array}{l}39.96 \\
40.26 \\
40.35 \\
40.57 \\
40.61 \\
40.22 \\
40.49 \\
40.67 \\
\end{array}$ & $\begin{array}{c}64 \text { / } 68 \\
97 / 148 \\
113 / 169 \\
110 / 162 \\
150 / 161 \\
86 / 111 \\
242 \text { / } 246 \\
285 / 261 \\
\end{array}$ \\
\hline NGC 5846 & $\begin{array}{c}0-0.5 \\
0.5-1 \\
1-1.5 \\
1.5-2 \\
2-3 \\
3-4 \\
4-8\end{array}$ & $\begin{array}{l}0.659_{-0.008}^{+0.009} \\
0.621_{-0.012}^{+0.012} \\
0.636_{-0.018}^{+0.018} \\
0.682_{-0.019}^{+0.022} \\
0.811_{-0.013}^{+0.013} \\
1.060_{-0.051}^{+0.120} \\
1.057_{-0.037}^{+0.020}\end{array}$ & $\begin{array}{c}0.48_{-0.08}^{+0.08} \\
- \\
- \\
- \\
- \\
-\end{array}$ & $\begin{array}{c}0.45_{-0.17}^{+0.11} \\
- \\
- \\
- \\
- \\
-\end{array}$ & $\begin{array}{c}0.52_{-0.03}^{+0.02} \\
- \\
- \\
- \\
- \\
-\end{array}$ & $\begin{array}{l}40.89 \\
40.96 \\
40.89 \\
40.97 \\
41.05 \\
40.65 \\
40.83\end{array}$ & $\begin{array}{l}39.83 \\
39.66 \\
39.75 \\
39.79 \\
39.81 \\
39.89 \\
40.66\end{array}$ & $\begin{array}{c}351 / 359 \\
- \\
- \\
- \\
- \\
-\end{array}$ \\
\hline
\end{tabular}

${ }^{a}$ The inner and outer radii to integrated the spectrum.

${ }^{b} k T$ is ISM temperature. $L_{\mathrm{ISM}}$ and $L_{\text {hard }}$ are the X-ray luminosities of the thermal emission and the non thermal emission in the range of $0.3-$ $2.0 \mathrm{keV}$, respectively.

${ }^{c}$ We fitted the spectra with a $2 \mathrm{~T}$ model.

${ }^{*}$ Fixed to the value. 
R. Nagino and K. Matsushita: Gravitational potential and X-ray luminosities of early-type galaxies, Online Material p 5
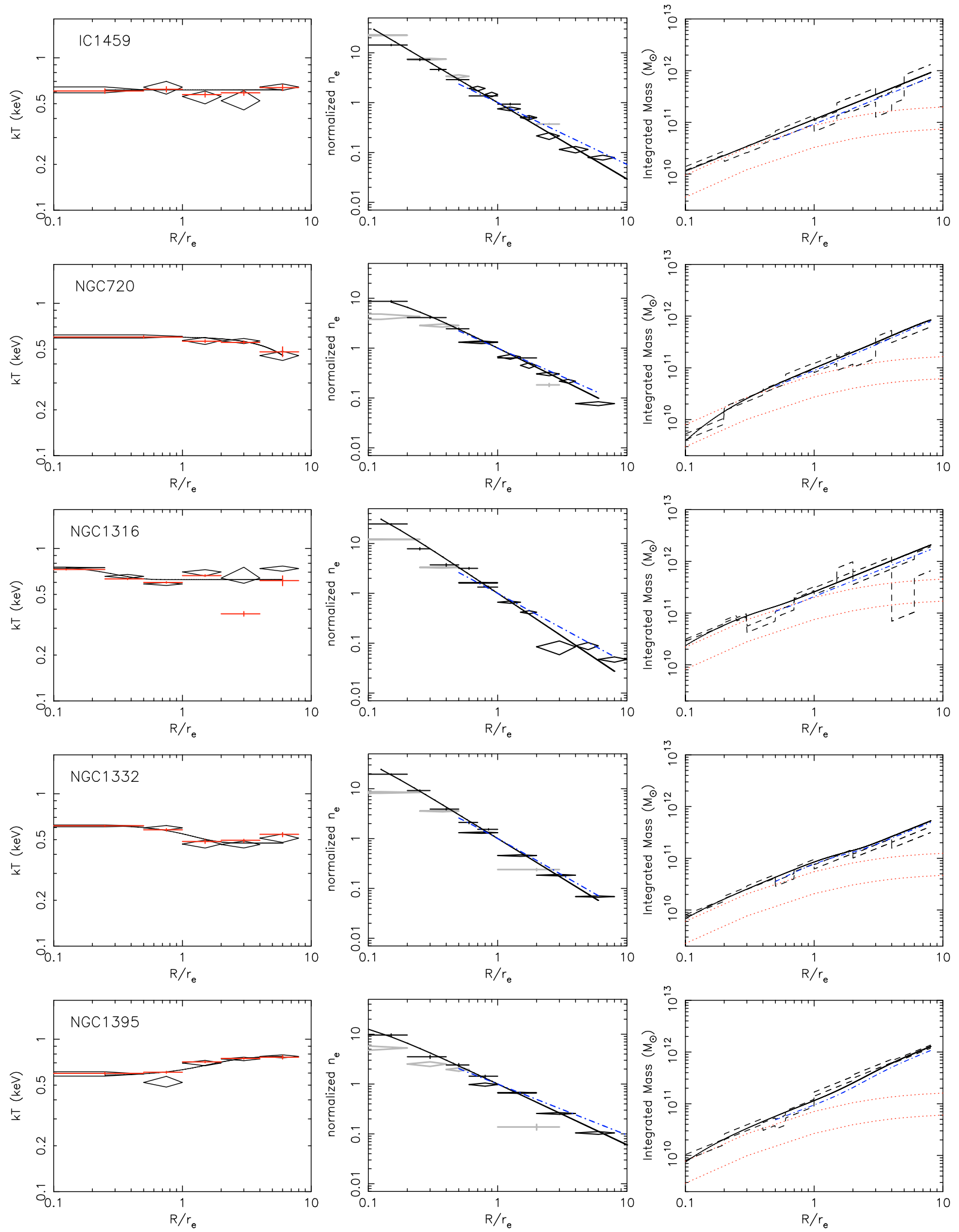

Fig. 18. (Left) The ISM temperature profiles of sample galaxies. The cross data points represent temperature profile derived from projected spectra, and the diamond data points are from deprojected spectra. The solid lines represent each best-fit function. (Center) The ISM density profiles. The diamond data points represent XMM-Newton data, and the cross data points are Chandra data obtained from spatial analysis. The solid lines represent each best-fit function. The black data points are used to fit the function, but the gray data points are not. The dot-dashed lines show best-fit function of the ISM density profile obtained from the spectral fit. These ISM density profiles are normalized by the value at $1 r_{\mathrm{e}}$. (Right) The integrated mass profiles. The solid and dot-dashed lines are total mass obtained from ISM density profiles from the surface brightness and spectral fit, respectively. The dashed lines are upper and lower limit. We also plotted the stellar mass profiles assuming stellar $M / L_{B}$ to be 3 and 8 (dotted lines). 
R. Nagino and K. Matsushita: Gravitational potential and X-ray luminosities of early-type galaxies, Online Material p 6
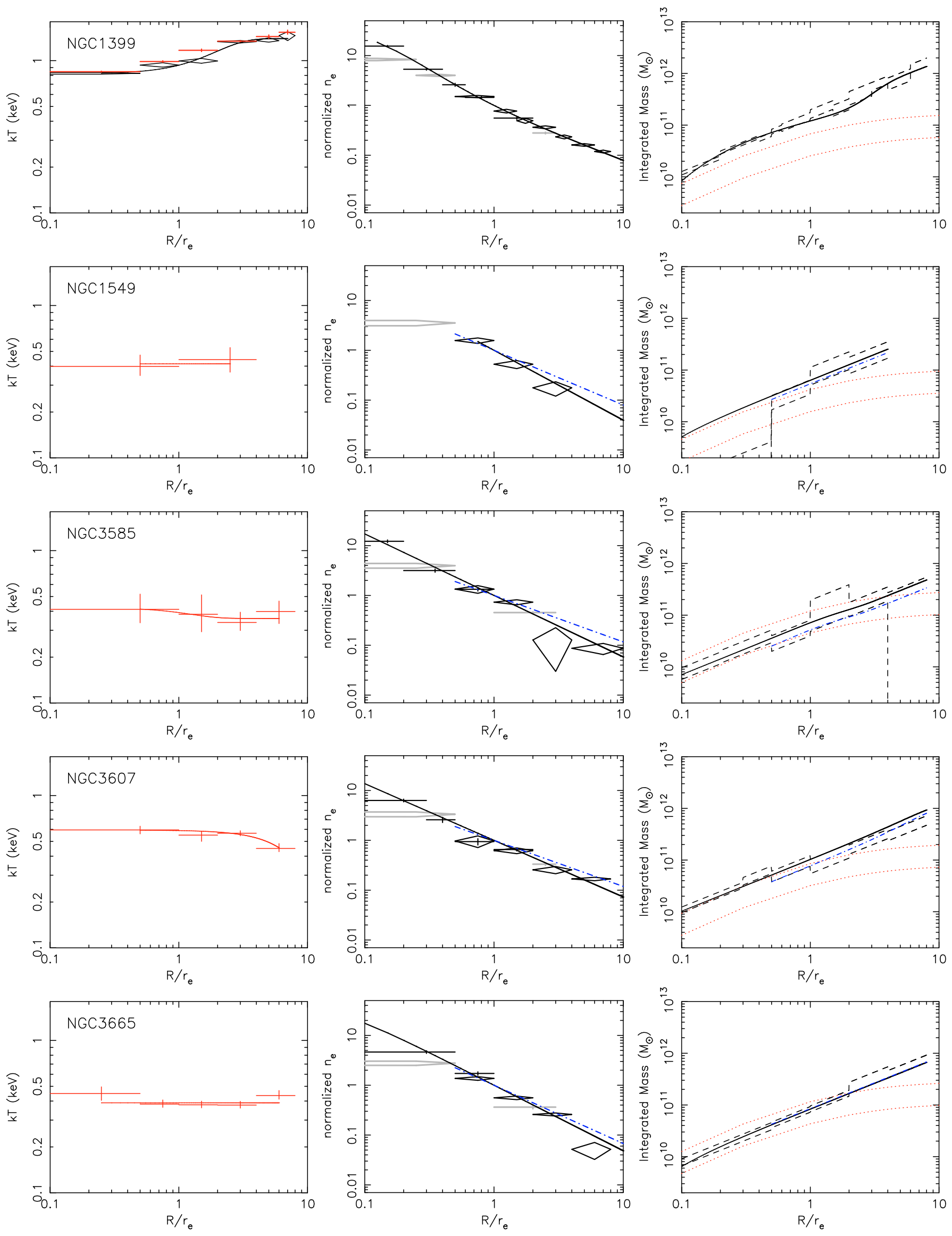

Fig. 18. continued. 
R. Nagino and K. Matsushita: Gravitational potential and X-ray luminosities of early-type galaxies, Online Material p 7
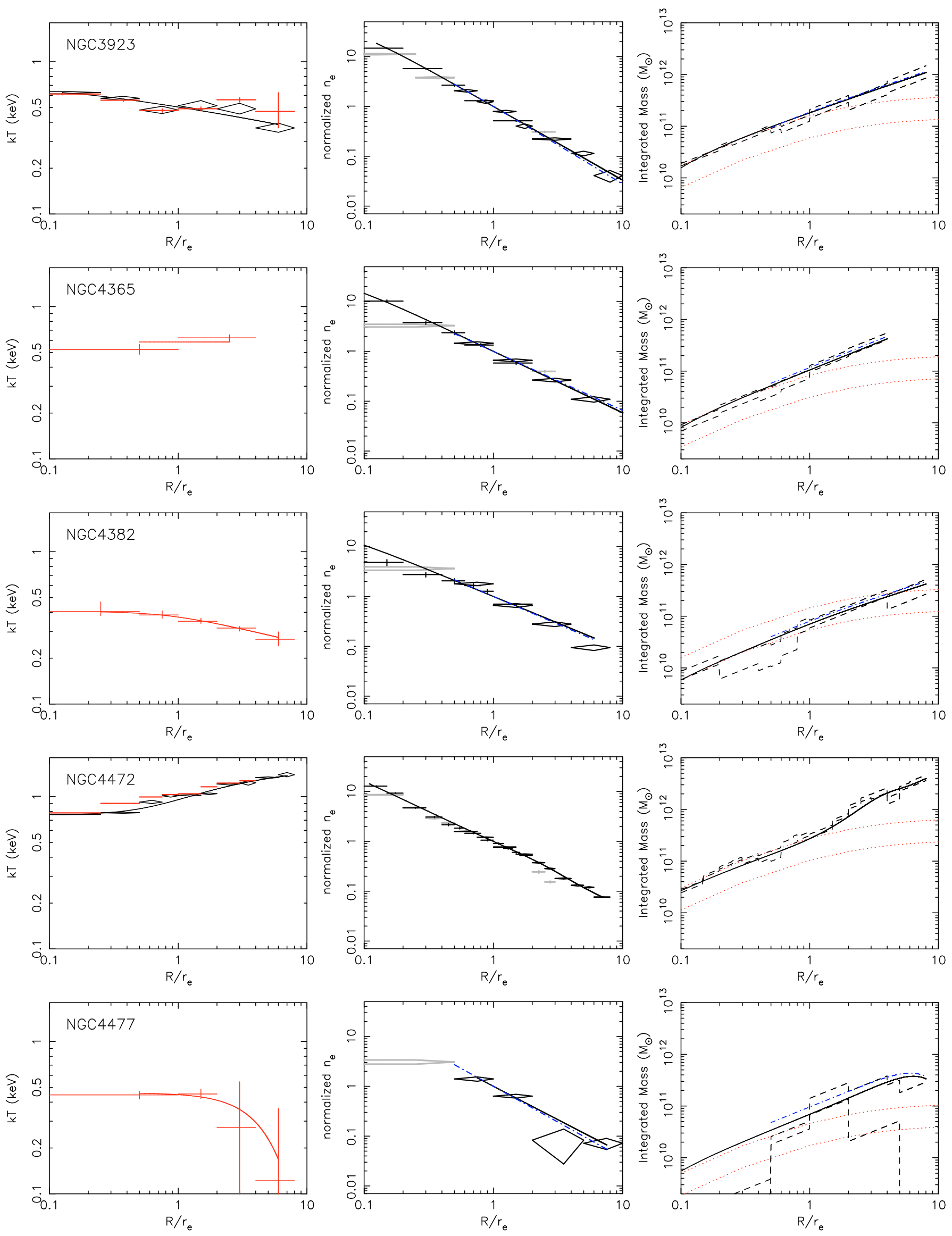

Fig. 18. continued. 
R. Nagino and K. Matsushita: Gravitational potential and X-ray luminosities of early-type galaxies, Online Material p 8
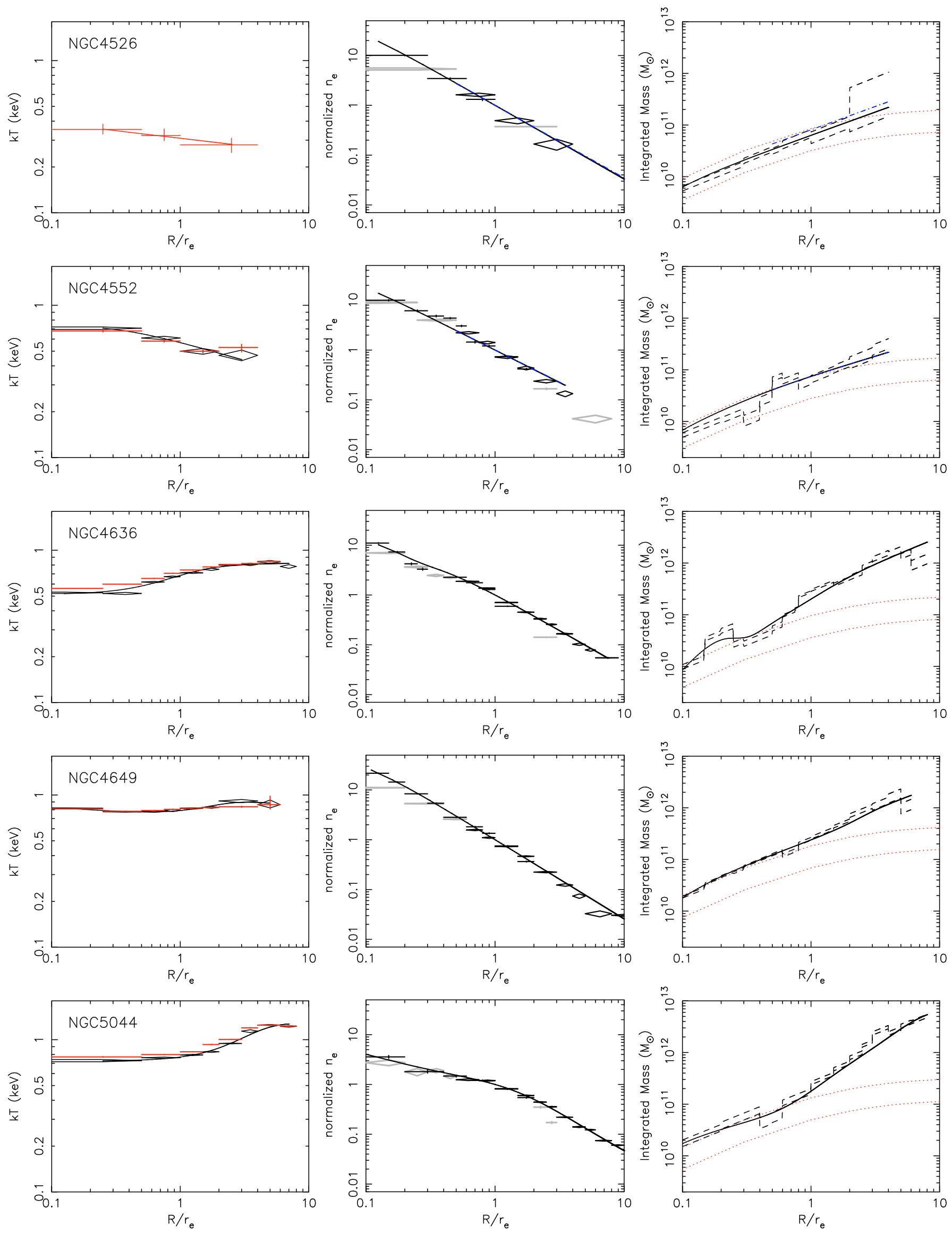

Fig. 18. continued. 
R. Nagino and K. Matsushita: Gravitational potential and X-ray luminosities of early-type galaxies, Online Material p 9
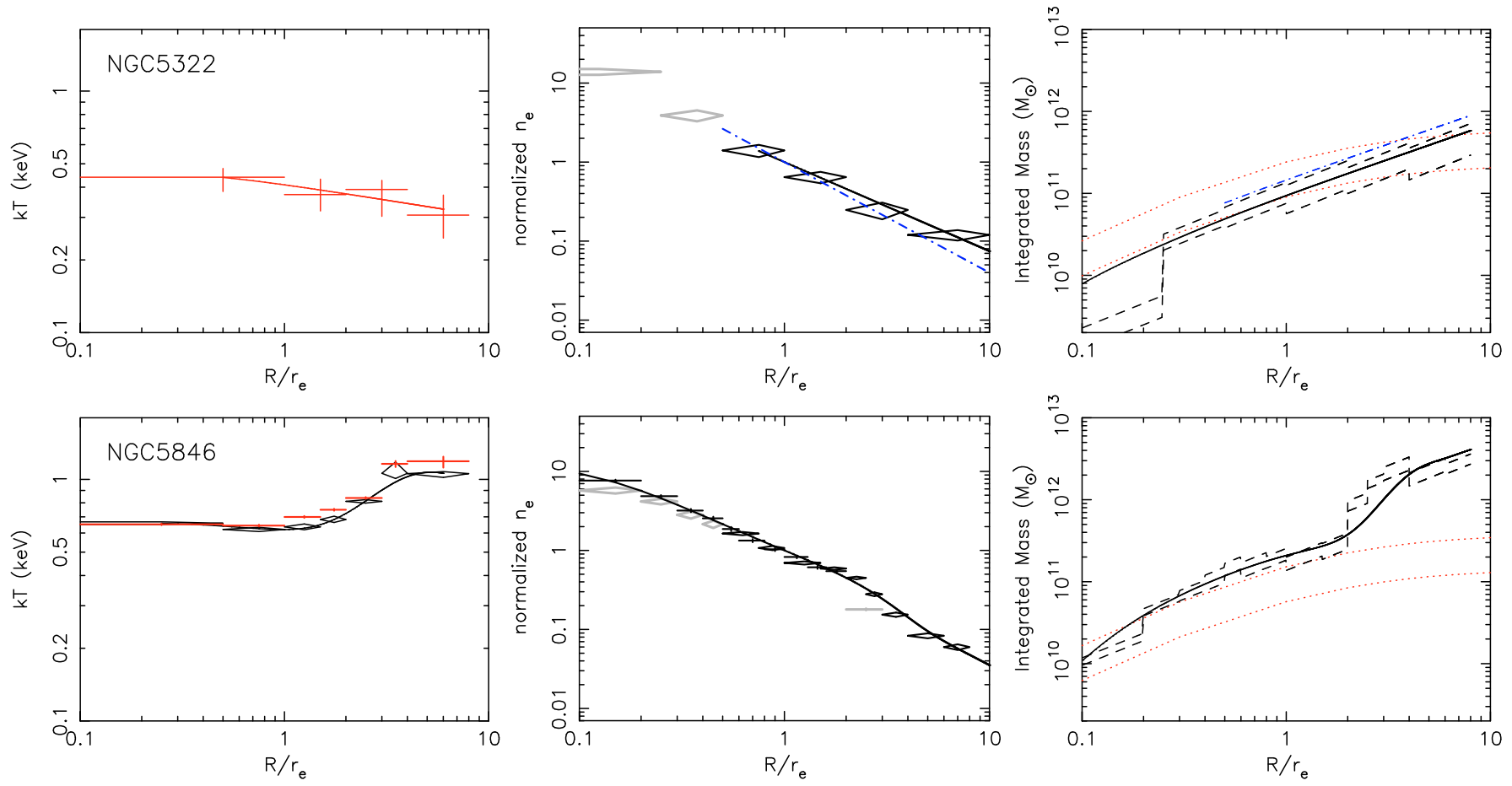

Fig. 18. continued. 